\title{
Distributed Continuous-time Approximate Projection Protocols for Shortest Distance Optimization Problems*
}

\author{
Youcheng Lou, Yiguang Hong, Shouyang Wang
}

\begin{abstract}
In this paper, we investigate the distributed shortest distance optimization problem for a multi-agent network to cooperatively minimize the sum of the quadratic distances from some convex sets, where each set is only associated with one agent. To deal with the optimization problem with projection uncertainties, we propose a distributed continuous-time dynamical protocol based on a new concept of approximate projection. Here each agent can only obtain an approximate projection point on the boundary of its convex set, and communicate with its neighbors over a time-varying communication graph. First, we show that no matter how large the approximate angle is, the system states are always bounded for any initial condition, and uniformly bounded with respect to all initial conditions if the inferior limit of the stepsize is greater than zero. Then, in the two cases, nonempty intersection and empty intersection of convex sets, we provide stepsize and approximate angle conditions to ensure the optimal convergence, respectively. Moreover, we give some characterizations about the optimal solutions for the empty intersection case and also present the convergence error between agents' estimates and the optimal point in the case of constant stepsizes and approximate angles.
\end{abstract}

Keywords: distributed optimization; convex intersection; shortest distance optimization; approximate projection

\section{Introduction}

In recent years, distributed optimization of a sum of convex functions has attracted much attention due to its wide applications in resource allocation, source localization, and robust

*This work was supported by the National Natural Science Foundation of China under Grant 71401163 and 61333001, Beijing Natural Science Foundation 4152057, Hong Kong Research Grants Council under Grant 419511 and Hong Kong Scholars Program under Grant XJ2015049. 
estimation (referring to [5, 19, 20, 21, 23, 28, 29, 30]). A whole optimization task can be accomplished cooperatively by a group of autonomous agents via simple local information exchange and distributed protocol design even when the communication graph among agents is time-varying.

Although many existing distributed optimization works have been done by discretetime algorithms, more and more attention has been paid to continuous-time algorithms in recent years $[18,31,32,33,34,35,36]$, partially because the continuous-time models can be studied by various well-developed continuous-time methods or make the algorithms easily implemented in physical systems. A distributed continuous-time computation model was proposed to solve an optimization problem for a fixed undirected graph in [31], with the optimization achieved by controlling the sum of subgradients of convex functions to make the state enter the optimal solution set, and later this model was generalized to the weight balanced graph case in [33], for differentiable objective functions with globally Lipschitz continuous gradient. Another continuous-time distributed algorithm with constant stepsize was developed in [35] for optimization problems with positivity constraints in a fixed undirected graph case, where a lower bound of convergence rate and an upper bound on the agents' estimate error were presented. Moreover, the relationship between the existing dual decomposition and consensus-based methods for distributed optimization was revealed in [36], where both approaches were based on the subgradient method, but one with a proportional control term and the other with an integral control term.

When the optimal solution sets of agents' individual objective functions have a nonempty intersection, the distributed optimization problem is equivalent to the convex intersection problems (CIP) [11, 12, 14, 15, 17, 18, 20]. A projected consensus algorithm was proposed in [20] for a network to solve the CIP, and the authors showed that all agents converge to a common point in the intersection set for weight-balanced and jointly connected communication graphs. Later, a continuous-time dynamical system was designed and connectivity conditions were discussed for the optimal convergence in [18]. In addition, a random sleep algorithm was proposed with providing conditions to converge almost surely to a common point in the intersection set in [14], where agents randomly take the neighbor-based average or projection onto their individual sets based on a Bernoulli process. Almost all the existing optimization results were obtained based on the assumption that the exact projection point onto the convex sets can be obtained [11, 12, 17, 18, 20, 22, 27].

On the other hand, the intersection of the considered convex sets may be empty in practice. In this case, how to seek a point with the shortest (quadratic) distance to these sets is also important. For instance, the supply center location problem is concerned with how to seek the location of raw materials supply center so that the average transportation cost from the supply center to the multiple factories is minimal $([9,10])$; the source 
localization in a sensor network is related to estimate the location of the source emitting a signal based on the received signals of multiple sensors in a noisy environment $([4,27])$. In fact, the problem for both the empty and nonempty intersection case is referred to as the shortest distance optimization problem (SDOP). Obviously, CIP is a special case of SDOP, and the average consensus problem is also a special case of SDOP since the optimal solution of the minimum of the sum of quadratic functions from some points is exactly the average of these points. Some distributed algorithms were proposed to discuss SDOP. For example, [27] formulated the source localization problem as the SDOP in a plane and proposed a discrete-time distributed algorithm, with the adjacency matrices of communication graphs required to be doubly stochastic. Moreover, [22] proposed two distributed continuous-time algorithms to solve SDOP in the empty intersection case for connected graphs: the first one was designed for optimal consensus based on sign functions, and the second one was modified to avoid chattering but only to achieve the optimal consensus approximately.

The objective of this paper is to design a continuous-time distributed protocol to solve SDOP based on approximate projection. Note that the exact projection is usually hard to obtain in practice. Therefore, approximate projection may have to be discussed in different situations, and, in fact, [15] proposed a discrete-time approximate projected consensus algorithm to solve CIP. The motivation of the current research aims at analysis and distributed design to cooperatively solve SDOP with projection uncertainties and continuous-time dynamics. For example, in a practical robotic network to solve the SDOP, a continuous-time robot may not always obtain the exact projection point of its own convex set, but only spot some point on the set surface near the exact projection point. The contribution of this paper can be summarized as follows.

- We propose a new concept of approximate projection, which is related to some points on the convex set's boundary surface and close to the exact projection point when the exact projection is hard to obtain. In other words, we consider an approximate projection related to set boundary surfaces, different from that defined in a "triangle" in [15]. To overcome the analysis difficulties resulting from this new approximate projection, we employ a geometric method to convert the original problem to a heterogeneous stepsize problem.

- Given any approximate angle, we show that, with the proposed continuous-time algorithm, the agent states are always bounded for any initial condition, and uniformly bounded with respect to all initial conditions if the stepsize is not too small. The result with respect to the continuous-time algorithm is different from some results based on some discrete-time ones. In fact, $\pi / 4$ was shown to be the critical approximate angle for the boundedness of the discrete-time algorithm with the 
approximate projection defined in a triangle in [15].

- We study SDOP in both the nonempty and empty intersection cases, and propose a unified protocol based on the approximate projection. In fact, the proposed convergence conditions and proofs in the two cases are quite different. Note that our result is different from that in [22] because we handle approximate projections without assuming that the communication graph is always connected, and ours tackles both the nonempty and empty intersection cases, while [15] only does the nonempty intersection case. Moreover, we also discuss the convergence error between agents' estimates and the optimal point in the case of constant stepsizes and approximate angles. Our results are certainly consistent with those discrete-time algorithms in the literature such as $[19,20]$ based on the exact projection.

The paper is organized as follows. Section 2 shows some basic concepts and preliminary results. Section 3 defines an approximate projection concept and formulates our shortest distance optimization problem (SDOP), followed by Section 4 for the discussions on boundedness and stepsizes. Section 5 presents the main convergence results for the nonempty intersection case, while Section 6 for the empty intersection case. Section 7 discusses the constant stepsize and approximate angle case. Then Section 8 provides numerical simulations. Finally, Section 9 gives some concluding remarks.

Notations: $\otimes$ denotes the Kronecker product; 1 denotes the vector with all ones; $(A)_{i j}$ denotes the $i$-th row and $j$-th column entry of matrix $A ; y^{T}$ denotes the transpose of a vector $y \in \mathbb{R}^{m} ;|y|$ denotes the Euclidean norm of $y ;[v, z]$ denotes the line segment connecting the two points $v, z$; line $(v, z)$ denotes the line passing the two points $v, z$; for a set $K \subseteq \mathbb{R}^{m}$, $\operatorname{int}(K)$ and $\operatorname{bd}(K)=K \backslash \operatorname{int}(K)$ denote the sets of interior points and boundary points of $K$, respectively; for a closed convex set $K \subseteq \mathbb{R}^{m}, P_{K}(\cdot)$ denotes the projection operator onto $K ;|y|_{K}:=\left|y-P_{K}(y)\right|$ denotes the distance between $y$ and $K$; $\langle\cdot, \cdot\rangle$ denotes the Euclidean inner product in $\mathbb{R}^{m}$; the angle between nonzero vectors $y$ and $z$ is denoted as $\angle(y, z) \in[0, \pi]$, where $\cos \angle(y, z)=\langle y, z\rangle /(|y||z|) ; \operatorname{span}\left\{v_{1}, \ldots, v_{p}\right\}$ (aff $\left\{v_{1}, \ldots, v_{p}\right\}$ ) denotes the subspace (affine hull) generated by vectors $v_{1}, \ldots, v_{p}$.

\section{Preliminaries}

In this section, we give preliminaries on graph theory [1], convex analysis [2], the consensus model with disturbances [26]. 


\subsection{Graph Theory}

A multi-agent network can be described by a directed graph $\mathcal{G}=(\mathcal{V}, \mathcal{E})$, where $\mathcal{V}=$ $\{1,2, \ldots, n\}$ is the node (or agent) set and $\mathcal{E} \subseteq \mathcal{V} \times \mathcal{V}$ the arc set with the arc $(j, i) \in \mathcal{E}$ describing that node $i$ can receive the information of node $j$. Here $(i, i) \notin \mathcal{E}$ for all $i$. Let $\mathcal{N}_{i}=\{j \in \mathcal{V} \mid(j, i) \in \mathcal{E}\}$ be the set of neighbors of node $i$. A path from node $i$ to node $j$ in $\mathcal{G}$ is a sequence $\left(i, i_{1}\right),\left(i_{1}, i_{2}\right), \ldots,\left(i_{p}, j\right)$ of $\operatorname{arcs}$ in $\mathcal{E}$. Graph $\mathcal{G}$ is said to be strongly connected if there exists a path from $i$ to $j$ for each pair of nodes $i, j \in \mathcal{V}$. Graph $\mathcal{G}$ is undirected when $(j, i) \in \mathcal{E}$ if and only if $(i, j) \in \mathcal{E}$.

The communication over the network under consideration is switching and characterized by a directed graph process $\mathcal{G}_{\sigma(t)}=\left(\mathcal{V}, \mathcal{E}_{\sigma(t)}\right), t \geq 0$, with $\mathcal{E}_{\sigma(t)}$ the arc set of the graph at time $t$. Here $\sigma:[0, \infty) \rightarrow \mathcal{Q}$ is a piecewise constant function to describe the time-varying graph process, where $\mathcal{Q}$ is the index set of all possible graphs on $\mathcal{V}$. Let $\Delta:=\left\{t_{k}, k \geq 0\right\}$ with $t_{0}=0$ denote the set of all switching moments of switching graph $\mathcal{G}_{\sigma}$. As usual, we assume there is a dwell time $\tau>0$ between two consecutive graph switching moments, i.e., $t_{k+1}-t_{k} \geq \tau$ for all $k$. The switching graph $\mathcal{G}_{\sigma}$ is uniformly jointly strongly connected (UJSC) if there exists $T>0$ such that the union graph $\left(\mathcal{V}, \cup_{t \leq s<t+T} \mathcal{E}(s)\right)$ is strongly connected for $t \geq 0$.

\subsection{Convex Analysis}

A set $K \subseteq \mathbb{R}^{m}$ is convex if $\lambda z_{1}+(1-\lambda) z_{2} \in K$ for any $z_{1}, z_{2} \in K$ and $0<\lambda<1$. For a closed convex set $K$ in $\mathbb{R}^{m}$, we can associate with any $z \in \mathbb{R}^{m}$ a unique element $P_{K}(z) \in K$ satisfying $\left|z-P_{K}(z)\right|=\inf _{y \in K}|z-y|=:|z|_{K}$, where $P_{K}$ is called the projection operator onto $K$. We have the following properties for the projection operator $P_{K}$.

Lemma 2.1 Let $K$ be a closed convex set in $\mathbb{R}^{m}$. Then

(i) $\left\langle y-P_{K}(y), z-P_{K}(y)\right\rangle \leq 0$ for any $y$ and $z \in K$;

(ii) $\left|P_{K}(y)-z\right| \leq|y-z|$ for any $y \in \mathbb{R}^{m}$ and any $z \in K$;

(iii) $\left\langle y-P_{K}(y), z-y\right\rangle \leq|y|_{K}\left(|z|_{K}-|y|_{K}\right)$ for any $y$ and $z$;

(iv) $\left|P_{K}(y)-P_{K}(z)\right| \leq|y-z|$ for any $y$ and $z$.

Proof. (i) is an equivalent definition of convex projection; (ii) comes from Lemma 1 (b) in [20]. We now show (iii). First of all, $\left\langle y-P_{K}(y), P_{K}(z)-P_{K}(y)\right\rangle \leq 0$ by (i). It is also clear that $\left\langle y-P_{K}(y), z-P_{K}(z)\right\rangle \leq|y|_{K}|z|_{K}$. Then

$$
\begin{aligned}
\left\langle y-P_{K}(y), z-y\right\rangle & =\left\langle y-P_{K}(y), z-P_{K}(z)+P_{K}(z)-P_{K}(y)+P_{K}(y)-y\right\rangle \\
& \leq|y|_{K}|z|_{K}-|y|_{K}^{2} .
\end{aligned}
$$


Thus, the inequality (iii) follows. (iv) is the standard non-expansive property.

The following lemma characterizes the distance between convex sets and their nonempty intersection, which can be found from Proposition 5.6.1 on page 72 in [6].

Lemma 2.2 Let $K_{1}, \ldots, K_{n}$ be closed convex sets in $\mathbb{R}^{m}$. If $\bigcap_{i=1}^{n} \operatorname{int}\left(K_{i}\right) \neq \emptyset$, then for every bounded set $S$, there exists $\kappa_{S}>0$ such that

$$
|x|_{\bigcap_{i=1}^{n} K_{i}}^{2} \leq \kappa_{S} \max _{1 \leq i \leq n}|x|_{K_{i}}^{2}, \forall x \in S
$$

The following lemma can be found from Proposition 1 on page 24 in [3].

Lemma 2.3 Let $K$ be a closed convex set in $\mathbb{R}^{m}$. Then $|x|_{K}^{2}$ is continuously differentiable and

$$
\nabla|x|_{K}^{2}=2\left(x-P_{K}(x)\right)
$$

A function $\varphi(\cdot): \mathbb{R}^{m} \rightarrow \mathbb{R}$ is said to be convex if $\varphi\left(\lambda z_{1}+(1-\lambda) z_{2}\right) \leq \lambda \varphi\left(z_{1}\right)+(1-$ $\lambda) \varphi\left(z_{2}\right)$ for any $z_{1}, z_{2} \in \mathbb{R}^{m}$ and $0<\lambda<1$, and it is $\ell$-strongly convex if $\varphi\left(\lambda z_{1}+(1-\lambda) z_{2}\right) \leq$ $\lambda \varphi\left(z_{1}\right)+(1-\lambda) \varphi\left(z_{2}\right)-\frac{1}{2} \ell \lambda(1-\lambda)\left|z_{1}-z_{2}\right|^{2}$ for any $z_{1}, z_{2} \in \mathbb{R}^{m}$ and $0<\lambda<1$. The following two inequalities hold for a continuously differentiable convex and $\ell$-strongly convex function $\varphi$, respectively:

$$
\begin{aligned}
& \varphi(y) \geq \varphi(x)+\langle y-x, \nabla \varphi(x)\rangle, \forall x, y \in \mathbb{R}^{m}, \\
& \varphi(y) \geq \varphi(x)+\langle y-x, \nabla \varphi(x)\rangle+\frac{\ell}{2}|y-x|^{2}, \forall x, y \in \mathbb{R}^{m} .
\end{aligned}
$$

The upper Dini derivative of function $g:(a, b) \rightarrow \mathbb{R}$ at $t \in(a, b)$ is defined as

$$
D^{+} g(t)=\limsup _{s \rightarrow 0^{+}} \frac{g(t+s)-g(t)}{s} .
$$

$g$ is non-increasing on $(a, b)$ if $D^{+} g(t) \leq 0, \forall t \in(a, b)$. The following result was shown in [8].

Lemma 2.4 Let $g_{i}(t, x): \mathbb{R} \times \mathbb{R}^{m} \rightarrow \mathbb{R}, i=1, \ldots, n$ be continuously differentiable and $g(t, x)=\max _{1 \leq i \leq n} g_{i}(t, x)$. Then $D^{+} g(t, x(t))=\max _{i \in \mathcal{I}(t)} \dot{g}_{i}(t, x(t))$ with $\mathcal{I}(t)=$ $\left\{i \mid g_{i}(t, x(t))=g(t, x(t)), 1 \leq i \leq n\right\}$.

\subsection{Consensus}

Consider the following consensus model with disturbance $w_{i}$,

$$
\dot{z}_{i}(t)=\sum_{j \in \mathcal{N}_{i}(t)}\left(z_{j}(t)-z_{i}(t)\right)+w_{i}(t), i=1, \ldots, n,
$$


where the disturbance $w_{i}(t):[0, \infty) \rightarrow \mathbb{R}$ is continuous. System (3) has a continuous solution, which satisfies (3) for almost all $t$ except at the switching moments of switching graph $\mathcal{G}_{\sigma}$. Consensus is said to be achieved for system (3) if for any initial condition, $\lim _{t \rightarrow \infty}\left|z_{i}(t)-z_{j}(t)\right|=0$ for all $1 \leq i, j \leq n$.

The next two lemmas can be obtained from the proofs of Theorem 4.2 and Proposition 4.10 in [26], respectively.

Lemma 2.5 If the switching graph $\mathcal{G}_{\sigma}$ is UJSC for system (3), then there exist $0<\beta<1$ and $B_{0}, B_{1}>0$ such that

$$
\begin{aligned}
H\left((k+1) B_{0}\right) & \leq \beta H\left(k B_{0}\right)+B_{1} \int_{k B_{0}}^{(k+1) B_{0}} \max _{1 \leq i \leq n}\left|w_{i}(t)\right| d t, \forall k \geq 0, \\
H(t) & \leq H\left(k B_{0}\right)+B_{1} \int_{k B_{0}}^{(k+1) B_{0}} \max _{1 \leq i \leq n}\left|w_{i}(t)\right| d t, \forall t: k B_{0} \leq t<(k+1) B_{0},
\end{aligned}
$$

where $H(t)=\max _{1 \leq i, j \leq n}\left|z_{i}(t)-z_{j}(t)\right|$.

Lemma 2.6 Suppose the switching graph $\mathcal{G}_{\sigma}$ of system (3) is UJSC and $\lim _{t \rightarrow \infty} w_{i}(t)=0$ for all $i$. Then consensus is achieved for system (3).

In the following consensus analysis, we need to extend the standard Barbalat's Lemma to switching cases. A function $g:[0, \infty) \rightarrow \mathbb{R}$ is uniformly continuous with respect to time intervals $\left(s_{k}, s_{k+1}\right), s \geq 0$ with $s_{k+1}-s_{k} \geq v$ for some $v>0$, if, for any $\varepsilon>0$, there is $\delta>0$, which depends on $\left\{s_{k}\right\}_{k \geq 0}$ and $g$, such that, for any $k$ and $r_{1}, r_{2}$ with $s_{k}<r_{1}<r_{2}<s_{k+1}$, $\left|g\left(r_{2}\right)-g\left(r_{1}\right)\right| \leq \varepsilon$ when $\left|r_{2}-r_{1}\right| \leq \delta$. We now introduce an extended Babalat's Lemma, which can be found in [16].

Lemma 2.7 For a continuous function $g$, suppose $\lim _{t \rightarrow \infty} g(t)=g_{0}$ exists and $g$ is continuously differential on each interval $\left(s_{k}, s_{k+1}\right)$, whose derivative $\dot{g}$ is uniformly continuous with respect to time intervals $\left(s_{k}, s_{k+1}\right)$ for $k \geq 0$. Then $\lim _{t \rightarrow \infty} \dot{g}(t)=0$.

\section{Approximate Projection and Problem Formulation}

In this section, we introduce the distributed SDOP and the distributed continuous-time approximate projected algorithm.

Consider a network of $n$ agents (or nodes) and bounded closed convex sets $X_{i} \subseteq \mathbb{R}^{m}$ for $i=1, \ldots, n$, with $X_{i}$ only associated with (or known by) agent $i$. The goal of the network is to cooperatively find a point $x^{*}$ with the shortest quadratic distance from the $n$ closed convex sets:

$$
x^{*} \in \arg \min f(x), \quad f(x)=\sum_{i=1}^{n}|x|_{X_{i}}^{2} .
$$


Projection-based methods have been widely adopted in the literature to solve CIP and constrained optimization problems, and almost all methods require that the exact projection can be obtained $[6,17,18,20,22,23,27]$. Since the exact projection may be difficult to obtain in practice, each agent may only obtain an approximate projection point located on the convex set surface and near the exact projection point. To be strict, we give the following definition.

Definition 3.1 Let $0 \leq \theta<\pi / 2$ and $K$ be a closed convex set in $\mathbb{R}^{m}$. Define sets

$$
\begin{aligned}
& \mathbf{C}_{K}(v, \theta)=v+\left\{\left.z\left|\left\langle z, P_{K}(v)-v\right\rangle \geq\right| z|| v\right|_{K} \cos \theta\right\}, \\
& \mathbf{b}(v, K)=\{z \mid z \in b d(K),[v, z] \cap b d(K)=\{z\}\} .
\end{aligned}
$$

The approximate projection $\mathbf{P}_{K}^{a}(v, \theta)$ of point $v$ onto $K$ is defined as the following set:

$$
\mathbf{P}_{K}^{a}(v, \theta)= \begin{cases}\mathbf{C}_{K}(v, \theta) \cap \mathbf{b}(v, K), & \text { if } v \notin K ; \\ \{v\}, & \text { otherwise. }\end{cases}
$$

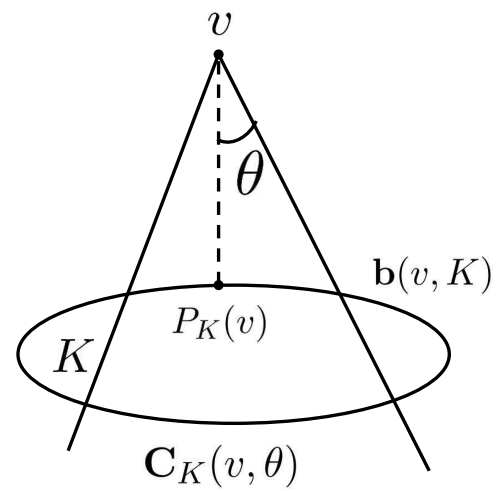

Figure 1: The approximate projection of point $v$ onto closed convex set $K$.

As shown in Fig. 1, the cone $\mathbf{C}_{K}(v, \theta)-v$ consists of all vectors having angle with the direction $P_{K}(v)-v$ less than $\theta$, and $\mathbf{b}(v, K)$ is the region on the boundary of $K$ that the agent can "see" starting from point $v$. Obviously, the exact projection $P_{K}(v) \in \mathbf{P}_{K}^{a}(v, \theta)$ for any $v \in \mathbb{R}^{m}$ and $0 \leq \theta<\pi / 2$ and $\mathbf{P}_{K}^{a}(v, 0)=\left\{P_{K}(v)\right\}$.

Remark 3.1 The approximate projection is more "practical" than the exact projection. For example, a robot likes to get its exact projection point on its convex target set when it approaches the set. However, in reality, it may select another point on the set surface as the exact one by mistake or to avoid expensive measurement or tedious computation. Then the selected projection point becomes an approximate one. In other words, this concept 
Lou et al. Distributed Continuous-time Approximate Projection Protocols

captures the situation when agents can only obtain some point on the set surface, which may not be but close to the exact projection point. Note that this concept is different from that given in [15], where the approximate projection point is located in a "triangle" region specified by $v$, the hyperplane of $K$ on $P_{K}(v)$ and the approximate angle $\theta$.

We next give some basic assumptions for our following analysis.

A1 (Connectivity) The switching graph $\mathcal{G}_{\sigma}$ is UJSC.

A2 (Convex Sets) (i) The boundary surfaces of convex sets $X_{i}, i=1, \ldots, n$ are regular (or smooth);

(ii) The convex set $X_{i}$ contains nonempty interior points for $i=1, \ldots, n$.

The definition of regularity or smoothness of a manifold can be easily found (referring to Definition 1 on page 52 in [7] for more details). Note that the Gaussian curvature of regular (or smooth) surfaces of closed bounded sets are bounded. In fact, A2 is quite mild. The boundaries of many well-known sets, such as the surfaces of spheres, ellipsoids, are regular; and moreover, the assumption that set $X_{i} \subseteq \mathbb{R}^{m}$ contains nonempty interior points is equivalent to $\operatorname{dim}\left(X_{i}\right)=m$ (where $\operatorname{dim}\left(X_{i}\right)$ denotes the dimension of the affine hull of set $X_{i}$ ), which was also widely used in the literature.

Let $P_{X_{i}}^{a}(\cdot): \mathbb{R}^{m} \rightarrow \mathbb{R}^{m}$ be a continuous map with $P_{X_{i}}^{a}(v) \in \mathbf{P}_{X_{i}}^{a}\left(v, \theta_{i}(v)\right)$ for any $v$, where

$$
\theta_{i}(v)=\angle\left(P_{X_{i}}^{a}(v)-v, P_{X_{i}}(v)-v\right)
$$

$0 \leq \theta_{i}(v)<\pi / 2$. Let $\theta_{i}(v)=0$ for simplicity when $v \in X_{i}$. In this paper, $\theta_{i}(v)$ is referred to as the approximate angle of $v$ onto $X_{i}$. The following assumption was used in Lou et al. (2014).

A3 (Approximate Angle) There exists $0<\theta^{*}<\pi / 2$ such that $0 \leq \theta_{i}(v) \leq \theta^{*}$ for all $i, v$.

Here we propose a distributed continuous-time approximate projected algorithm:

$$
\dot{x}_{i}(t)=\sum_{j \in \mathcal{N}_{i}(t)}\left(x_{j}(t)-x_{i}(t)\right)+\alpha_{t}\left(P_{X_{i}}^{a}\left(x_{i}(t)\right)-x_{i}(t)\right), i=1, \ldots, n,
$$

where $x_{i} \in \mathbb{R}^{m}$ is the state estimate of agent $i$ for the optimal solution, $\mathcal{N}_{i}(t)$ is the neighbor set of node $i$ at time $t,\left\{\alpha_{t}\right\}$ is the stepsize $\left(0 \leq \alpha_{t} \leq \alpha^{*}, \alpha^{*}>0\right)$ and is uniformly continuous over $t$. The continuity of stepsize $\alpha_{t}$ and maps $P_{X_{i}}^{a}(\cdot)$ guarantees that $(5)$ has a solution that is continuous over $[0, \infty)$ and continuously differentiable except at the switching moments of switching graph $\mathcal{G}_{\sigma}$.

Remark 3.2 The term $P_{X_{i}}^{a}(x)-x$ can be viewed as a negative "approximate" gradient because it becomes the negative gradient of $\frac{1}{2}|x|_{X_{i}}^{2}$ by noting that $\nabla|x|_{X_{i}}^{2}=2\left(x-P_{X_{i}}(x)\right)$ in the exact projection case (i.e., $P_{X_{i}}^{a}\left(x_{i}(t)\right)=P_{X_{i}}\left(x_{i}(t)\right)$ ). In fact, (5) with taking $\alpha_{t} \equiv 1$ and exact projection was proposed in [18] to solve the CIP. 
The convergence analysis of (5) is not easy because the gradient term is corrupted with state-dependent approximation and there is no explicit expression to describe the relationship between the approximate projection point and the exact one. To handle the problem, we make some transformation. In the case of $v \notin X_{i}$, we define by $P_{X_{i}}^{h}(v)$ the intersection point of the hyperplane of $X_{i}$ at $P_{X_{i}}(v)$ (the tangent plane of $\operatorname{bd}\left(X_{i}\right)$ at $\left.P_{X_{i}}(v)\right)$ with $P_{X_{i}}(v)-v$ as the normal direction and the line segment $\left[v, P_{X_{i}}^{a}(v)\right]$, as shown in Fig. 2. Clearly, $P_{X_{i}}^{h}(v)=P_{X_{i}}(v)$ when $P_{X_{i}}^{a}(v)=P_{X_{i}}(v)$. In the case of $v \in X_{i}$, we define $P_{X_{i}}^{h}(v)=P_{X_{i}}^{a}(v)=v$. Then we can find that $P_{X_{i}}^{h}(v)=v$ if and only if $v \in X_{i}$. We write

$$
P_{X_{i}}^{a}(v)-v=\gamma_{X_{i}}(v)\left(P_{X_{i}}^{h}(v)-v\right),
$$

where $\gamma_{X_{i}}(v)=\frac{\left|P_{X_{i}}^{a}(v)-v\right|}{\left|P_{X_{i}}^{h}(v)-v\right|} \geq 1$ if $P_{X_{i}}^{h}(v) \neq v$, and $\gamma_{X_{i}}(v)=1$ otherwise.

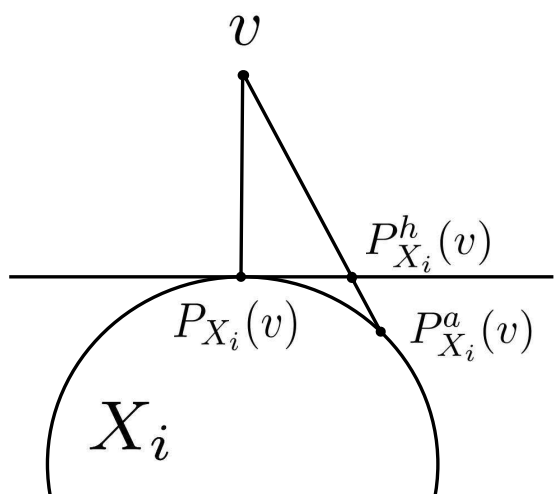

Figure 2: An illustration for $P_{X_{i}}^{h}(v)$.

Rewrite $\alpha_{t}\left(P_{X_{i}}^{a}\left(x_{i}(t)\right)-x_{i}(t)\right)=\alpha_{i, t}\left(P_{X_{i}}^{h}\left(x_{i}(t)\right)-x_{i}(t)\right)$, with the virtual stepsize of agent $i$ defined as

$$
\alpha_{i, t}= \begin{cases}\gamma_{X_{i}}\left(x_{i}(t)\right) \alpha_{t}=\frac{\left|P_{X_{i}}^{a}\left(x_{i}(t)\right)-x_{i}(t)\right|}{\left|P_{X_{i}}^{h}\left(x_{i}(t)\right)-x_{i}(t)\right|} \alpha_{t}, & \text { if } P_{X_{i}}^{h}\left(x_{i}(t)\right) \neq x_{i}(t) \\ \alpha_{t}, & \text { otherwise. }\end{cases}
$$

Clearly, $\alpha_{i, t} \geq \alpha_{t}$. Although the designed stepsize $\alpha_{t}$ is the same for all agents, agent $i$ has its own virtual stepsize $\alpha_{i, t}$ based on its own approximate projection. We express (5) in another form with heterogeneous virtual stepsizes:

$$
\dot{x}_{i}(t)=\sum_{j \in \mathcal{N}_{i}(t)}\left(x_{j}(t)-x_{i}(t)\right)+\alpha_{i, t}\left(P_{X_{i}}^{h}\left(x_{i}(t)\right)-x_{i}(t)\right), i=1, \ldots, n .
$$

Then we give a definition for our problem as follows. 
Definition 3.2 The shortest distance optimization problem (SDOP) is solved by (5) or (6) if, for any initial condition $x_{i}(0) \in \mathbb{R}^{m}, i=1, \ldots, n$, there exists $x^{*} \in \arg \min \sum_{i=1}^{n}|x|_{X_{i}}^{2}$ such that

$$
\lim _{t \rightarrow \infty} x_{i}(t)=x^{*}, i=1, \ldots, n .
$$

In the following three sections, we first establish some basic results, and then present the convergence results in the nonempty intersection and empty intersection cases.

\section{Discussions on Boundedness and Stepsizes}

In this section, we show the state boundedness and establish an "equivalent" relationship between the designed stepsize $\alpha_{t}$ and the virtual stepsize $\alpha_{i, t}$.

\subsection{Boundedness of System States}

Denote $\theta_{i, t}=\theta_{i}\left(x_{i}(t)\right)$ for simplicity. Note that $\theta_{i, t}$ is also equal to $\angle\left(P_{X_{i}}^{h}\left(x_{i}(t)\right)-\right.$ $\left.x_{i}(t), P_{X_{i}}\left(x_{i}(t)\right)-x_{i}(t)\right)$. Here we study the boundedness of $x_{i}(t), i \in \mathcal{V}, t \geq 0$ of $(6)$ with the approximate angle $\theta_{i, t}$.

Let $X_{c}=\operatorname{co}\left\{X_{i}, i=1, \ldots, n\right\}$ be the convex hull of the sets $X_{i}, i=1, \ldots, n, \xi:=$ $\sup _{z_{1}, z_{2} \in X_{c}}\left|z_{1}-z_{2}\right|$, which is finite due to the boundedness of $X_{i}$ s.

Theorem 4.1 (i) For any initial condition $x_{i}(0), i \in \mathcal{V}$, the system states $x_{i}(t), i \in \mathcal{V}, t \geq$ 0 are bounded;

(ii) Suppose $\liminf _{t \rightarrow \infty} \alpha_{t}>0$. Then, for any $0<\theta<\pi / 2$ and any initial condition $x_{i}(0), i \in \mathcal{V}$,

$$
\limsup _{t \rightarrow \infty}\left|x_{i}(t)\right|_{X_{c}} \leq \max \left\{\frac{\xi}{\sin \theta}, \xi\left(\tan \theta+\sqrt{(\tan \theta)^{2}+2 \tan \theta}\right)\right\} .
$$

Furthermore, if A3 holds, then for any initial condition $x_{i}(0), i \in \mathcal{V}$,

$$
\limsup _{t \rightarrow \infty}\left|x_{i}(t)\right|_{X_{c}} \leq \xi\left(\tan \theta^{*}+\sqrt{\left(\tan \theta^{*}\right)^{2}+2 \tan \theta^{*}}\right) .
$$

Remark 4.1 A discrete-time algorithm was proposed in [15] to solve CIP with approximate projection, where $\pi / 4$ was found to be a critical approximate angle ensuring the boundedness of system states in the case of $\alpha_{i, k} \equiv 1$ and $\theta_{i, k} \equiv \theta, 0 \leq \theta<\pi / 2$. To be specific, the states are uniformly bounded with respect to all initial conditions when $\theta<\pi / 4$ and unbounded for most all initial conditions when $\theta>\pi / 4$. Different from this critical approximate angle result, Theorem 4.1 shows that the continuous-time system states are always bounded for any initial condition no matter how large $\theta$ is, and moreover, the states are uniformly bounded for all initial conditions with fixing $\alpha_{i, t} \equiv 1$. 
Remark 4.2 Notice that the boundedness results in Theorem 4.1 do not require any connectivity of communication graph. Moreover, when the exact projection is obtained $\left(\theta_{i, t}=0\right)$, Theorem 4.1 (ii) implies that all agents converge to the convex hull spanned by all the convex sets, which is related to the target aggregation and leader-following problems [13, 24, 25].

Here we present a result for a simple case: there is only one node in the network. Its set is bounded and denoted as $X_{*}$. We denote the states of this node as $x_{*}(t), t \geq 0$ driven by the continuous-time approximate projected dynamical system:

$$
\dot{x}_{*}(t)=\alpha_{t}\left(P_{X_{*}}^{a}\left(x_{*}(t)\right)-x_{*}(t)\right),
$$

where $P_{X_{*}}^{a}\left(x_{*}(t)\right) \in \mathbf{P}_{X_{*}}^{a}\left(x_{*}(t), \theta_{*}\left(x_{*}(t)\right)\right)$. Noticing that $\left\langle x_{*}(t)-P_{X_{*}}\left(x_{*}(t)\right), P_{X_{*}}^{a}\left(x_{*}(t)\right)-\right.$ $\left.P_{X_{*}}\left(x_{*}(t)\right)\right\rangle \leq 0$, we have

$$
\frac{d\left|x_{*}(t)\right|_{X_{*}}^{2}}{d t}=2\left\langle x_{*}(t)-P_{X_{*}}\left(x_{*}(t)\right), \dot{x}_{*}(t)\right\rangle \leq-2 \alpha_{t}\left|x_{*}(t)\right|_{X_{*}}^{2}
$$

Then for any initial condition $x_{*}(0)$ and any stepsize $\left\{\alpha_{t}\right\},\left|x_{*}(t)\right|_{X_{*}} \leq\left|x_{*}(0)\right|_{X_{*}}$ always holds.

Now we present the proof of Theorem 4.1.

Proof. Let $t \notin \Delta$. Denote $\hbar_{i}(t)=\frac{1}{2}\left|x_{i}(t)\right|_{X_{c}}^{2}, \hbar(t)=\max _{1 \leq i \leq n} \hbar_{i}(t)$. By Lemmas 2.3 and 2.4, we have

$$
\begin{aligned}
D^{+} \hbar(t) & =\max _{i \in \mathcal{I}(t)}\left\langle x_{i}(t)-P_{X_{c}}\left(x_{i}(t)\right), \dot{x}_{i}(t)\right\rangle \\
& =\max _{i \in \mathcal{I}(t)}\left\langle x_{i}(t)-P_{X_{c}}\left(x_{i}(t)\right), \sum_{j \in \mathcal{N}_{i}(t)}\left(x_{j}(t)-x_{i}(t)\right)+\alpha_{i, t}\left(P_{X_{i}}^{h}\left(x_{i}(t)\right)-x_{i}(t)\right)\right\rangle,
\end{aligned}
$$

where $\mathcal{I}(t)=\left\{i \mid i \in \mathcal{V}, \hbar_{i}(t)=\hbar(t)\right\}$. Take $i \in \mathcal{I}(t)$. Lemma 2.1 (iii) implies that, for any $j$,

$$
\left\langle x_{i}-P_{X_{c}}\left(x_{i}\right), x_{j}-x_{i}\right\rangle \leq\left|x_{i}\right|_{X_{c}}\left(\left|x_{j}\right|_{X_{c}}-\left|x_{i}\right|_{X_{c}}\right) \leq 0 \text {. }
$$

According to Lemma 2.1 (i), $\left\langle x_{i}-P_{X_{c}}\left(x_{i}\right), P_{X_{i}}\left(x_{i}\right)-P_{X_{c}}\left(x_{i}\right)\right\rangle \leq 0$ due to $X_{i} \subseteq X_{c}$. Therefore,

$$
\left\langle x_{i}-P_{X_{c}}\left(x_{i}\right), P_{X_{i}}\left(x_{i}\right)-x_{i}\right\rangle \leq-\left|x_{i}\right|_{X_{c}}^{2}
$$

Moreover, recalling the definitions of $P_{X_{i}}^{h}\left(x_{i}(t)\right)$ and $\theta_{i, t}$, we have $\left\langle x_{i}(t)-P_{X_{i}}\left(x_{i}(t)\right), P_{X_{i}}^{h}\left(x_{i}(t)\right)-\right.$ 


$$
\begin{aligned}
&\left.P_{X_{i}}\left(x_{i}(t)\right)\right\rangle=0 \text { and }\left|P_{X_{i}}^{h}\left(x_{i}(t)\right)-P_{X_{i}}\left(x_{i}(t)\right)\right|=\tan \theta_{i, t}\left|x_{i}(t)\right| X_{i} \text {. Then } \\
&\left\langle x_{i}(t)-P_{X_{c}}\left(x_{i}(t)\right), P_{X_{i}}^{h}\left(x_{i}(t)\right)-P_{X_{i}}\left(x_{i}(t)\right)\right\rangle \\
&=\left\langle x_{i}(t)-P_{X_{i}}\left(x_{i}(t)\right)+P_{X_{i}}\left(x_{i}(t)\right)-P_{X_{c}}\left(x_{i}(t)\right), P_{X_{i}}^{h}\left(x_{i}(t)\right)-P_{X_{i}}\left(x_{i}(t)\right)\right\rangle \\
& \leq\left|P_{X_{i}}\left(x_{i}(t)\right)-P_{X_{c}}\left(x_{i}(t)\right)\right|\left|P_{X_{i}}^{h}\left(x_{i}(t)\right)-P_{X_{i}}\left(x_{i}(t)\right)\right| \\
& \leq \xi \tan \theta_{i, t}\left|x_{i}(t)\right|_{X_{i}} \\
& \leq \xi \tan \theta_{i, t}\left(\left|x_{i}(t)\right|_{X_{c}}+\xi\right),
\end{aligned}
$$

where the last inequality follows from the relation $\left|x_{i}\right|_{X_{i}} \leq\left|x_{i}-P_{X_{c}}\left(x_{i}\right)\right|+\mid P_{X_{c}}\left(x_{i}\right)-$ $\left.P_{X_{i}}\left(x_{i}\right)|\leq| x_{i}\right|_{X_{c}}+\xi$. Thus, based on (9) and (10), we have

$$
\left\langle x_{i}(t)-P_{X_{c}}\left(x_{i}(t)\right), P_{X_{i}}^{h}\left(x_{i}(t)\right)-x_{i}(t)\right\rangle \leq-\left|x_{i}(t)\right|_{X_{c}}^{2}+\xi \tan \theta_{i, t}\left(\left|x_{i}(t)\right|_{X_{c}}+\xi\right) .
$$

With (7), (8), (11) and $i \in \mathcal{I}(t)$, we obtain

$$
D^{+} \hbar(t) \leq \alpha_{i, t}\left(-2 \hbar(t)+\xi \tan \theta_{i, t}(\sqrt{2 \hbar(t)}+\xi)\right)
$$

We complete the proof by the following analysis.

(i) It is easy to see that for any $0<\hat{\theta}<\pi / 2, \theta_{i, t} \leq \hat{\theta}$ when $\left|x_{i}(t)\right|_{X_{c}} \geq \xi / \sin \hat{\theta}$. Then $D^{+} \hbar(t) \leq 0$ when $t \notin \Delta$ and

$$
\hbar(t) \geq \max \left\{\frac{\xi^{2}}{2(\sin \hat{\theta})^{2}}, \frac{\xi^{2}(\tan \hat{\theta})^{2}}{4}+\frac{\xi^{2} \tan \hat{\theta}}{2}\left(1+\frac{\sqrt{(\tan \hat{\theta})^{2}+4 \tan \hat{\theta}}}{2}\right)\right\} .
$$

Hence, system states are bounded for any initial condition.

(ii) Let $\alpha_{*}=\left(\liminf _{t \rightarrow \infty} \alpha_{t}\right) / 2>0$ and $\hat{t}$ be the moment such that when $t \geq \hat{t}$, $\alpha_{i, t} \geq \alpha_{t} \geq \alpha_{*}$. Then $D^{+} \hbar(t) \leq-\alpha_{*} \hbar(t)$ once $t \geq \hat{t}, t \notin \Delta$ and

$$
\hbar(t) \geq \max \left\{\frac{\xi^{2}}{2(\sin \theta)^{2}}, \xi^{2}(\tan \theta)^{2}+\xi^{2} \tan \theta\left(1+\sqrt{(\tan \theta)^{2}+2 \tan \theta}\right)\right\} .
$$

Therefore, $\hbar(t)$ is not greater than the number in the preceding inequality when $t \geq \hat{t}$ and $t \notin \Delta$. The second conclusion can be obtained directly based on the above arguments.

Thus, the conclusion follows from the continuity of $x_{i}(t)$.

\subsection{Equivalence between Stepsizes}

To obtain the convergence conditions, we establish a relationship between the designed stepsize $\alpha_{t}$ and virtual stepsizes $\alpha_{i, t}$. To show that they are equivalent in the sense that they can be bounded by each other, it suffices to establish the boundedness of $\gamma_{X_{i}}(\cdot)$.

Let $S=X_{c}+\mathbf{B}\left(0, r_{0}\right)$, where $\mathbf{B}\left(0, r_{0}\right)$ denotes the ball with center zero and radius $r_{0}>0$. Denote $\mu_{i}(v)=\angle\left(P_{X_{i}}(v)-P_{X_{i}}^{a}(v), v-P_{X_{i}}^{a}(v)\right)$. 
Lemma 4.1 If the map $P_{X_{i}}^{a}(\cdot)$ satisfies

$$
\inf _{v \in S \backslash X_{i}, P_{X_{i}}^{a}(v) \neq P_{X_{i}}(v)} \mu_{i}(v)>0,
$$

then $\sup _{v \in S} \gamma_{X_{i}}(v)<\infty$.

Its proof is in the Appendix. The following result provides a condition to guarantee the condition (12).

Lemma 4.2 Suppose A2 and A3 hold. Then (12) holds.

The proof is also in the Appendix. Because the states of (6) are bounded by Theorem 4.1, we take sufficiently large $r_{0}$ such that $S$ contains all the system states. We next show that $\mathbf{A} \mathbf{2}$ and $\mathbf{A} \mathbf{3}$ imply the equivalence between $\left\{\alpha_{t}\right\}$ and $\left\{\alpha_{i, t}\right\}$.

Clearly, $\alpha_{t}=\alpha_{i, t}$ when $x_{i}(t) \in X_{i}$ or $P_{X_{i}}^{a}\left(x_{i}(t)\right)=P_{X_{i}}\left(x_{i}(t)\right)$. Then we only need to focus on the case when $x_{i}(t) \notin X_{i}$ and $P_{X_{i}}^{a}\left(x_{i}(t)\right) \neq P_{X_{i}}\left(x_{i}(t)\right)$. By (39) in the Appendix, $\gamma_{X_{i}}\left(x_{i}(t)\right) \leq 1+\frac{1}{\sin \mu_{i, t}} \sin \theta_{i, t}$, where $\mu_{i, t}:=\mu_{i}\left(x_{i}(t)\right)$. Then we have

Theorem 4.2 Under A2 and A3,

$$
\alpha_{t} \leq \alpha_{i, t} \leq C_{i, t} \alpha_{t} \leq C_{i} \alpha_{t}, \forall t
$$

where $C_{i, t}=1+\frac{1}{\sin \mu_{i}} \sin \theta_{i, t}, C_{i}=1+\frac{1}{\sin \mu_{i}}$,

$$
\begin{aligned}
\mu_{i} & :=\inf _{t \geq 0, x_{i}(t) \notin X_{i}, P_{X_{i}}^{a}\left(x_{i}(t)\right) \neq P_{X_{i}}\left(x_{i}(t)\right)} \mu_{i, t} \\
& \geq \inf _{v \in S \backslash X_{i}, P_{X_{i}}^{a}(v) \neq P_{X_{i}}(v)} \mu_{i}(v)>0 .
\end{aligned}
$$

Note that the first inequality of (14) follows from $x_{i}(t) \in S$ and the second one from Lemma 4.2. In fact, (13) somehow characterizes the bounded bending property of smooth surfaces, which helps convert the convergence conditions on $\alpha_{i, t}$ into the conditions on $\alpha_{t}$.

Remark 4.3 As Theorem 4.2 shows, A2 and A3 guarantee the equivalence between the designed stepsize and the virtual stepsize. In fact, with (13), we found that under $\mathbf{A} \mathbf{1}$, the optimal convergence established in the next two sections hold for general convex sets (not necessary to satisfy A2 and A3). 


\section{Nonempty Intersection Case}

In this section, we show the convergence result in the nonempty intersection case, $\cap_{i=1}^{n} X_{i} \neq$ $\emptyset$. Clearly, $X_{0}:=\cap_{i=1}^{n} X_{i}$ is the optimal solution set of $\min \sum_{i=1}^{n}|x|_{X_{i}}^{2}$.

Theorem 5.1 Suppose A1-A3 hold and $\bigcap_{i=1}^{n} X_{i} \neq \emptyset$. Then SDOP is solved by system (6) if $\int_{0}^{\infty} \alpha_{t} d t=\infty, \int_{0}^{\infty} \alpha_{t} \tan \theta_{t}^{+} d t<\infty$. Furthermore, in the special case when $\theta_{i, t}=0 \forall i, t$, $S D O P$ is solved by (6) if and only if $\int_{0}^{\infty} \alpha_{t} d t=\infty$.

Remark 5.1 When the intersection set of all $X_{i} s$ is nonempty, SDOP (4) is equivalent to CIP of finding a point in $X_{0}[11,12,14,15,1 \%, 18,20,27]$. The optimal consensus algorithm based on the exact projection presented in [18] is a special case of (5) with taking $\alpha_{t} \equiv 1$ and $\theta_{i, t} \equiv 0$, which is consistent with Theorem 5.1. Theorem 5.1 is also consistent with the convex intersection computation results of discrete-time algorithms in [15, 20, 27].

Denote $\alpha_{t}^{+}=\max _{1 \leq i \leq n} \alpha_{i, t}, \theta_{t}^{+}=\max _{1 \leq i \leq n} \theta_{i, t}$, and the distance functions

$$
h(t)=\max _{1 \leq i \leq n} h_{i}(t), \quad h_{i}(t)=\frac{1}{2}\left|x_{i}(t)\right|_{X_{0}}^{2}, i=1, \ldots, n, t \geq 0 .
$$

Lemma 5.1 Suppose $\cap_{i=1}^{n} X_{i} \neq \emptyset$. Then $D^{+} h(t) \leq 2 \alpha_{t}^{+} \tan \theta_{t}^{+} h(t)$ for any $t \notin \Delta$.

Proof. Let $t \notin \Delta$. Similar to (7), we have

$$
D^{+} h(t)=\max _{i \in \mathcal{I}(t)}\left\langle x_{i}(t)-P_{X_{0}}\left(x_{i}(t)\right), \sum_{j \in \mathcal{N}_{i}(t)}\left(x_{j}(t)-x_{i}(t)\right)+\alpha_{i, t}\left(P_{X_{i}}^{h}\left(x_{i}(t)\right)-x_{i}(t)\right)\right\rangle,
$$

where $\mathcal{I}(t)=\left\{i \mid i \in \mathcal{V}, h_{i}(t)=h(t)\right\}$. Take $i \in \mathcal{I}(t)$. Similar to (8), we also have

$$
\left\langle x_{i}(t)-P_{X_{0}}\left(x_{i}(t)\right), x_{j}(t)-x_{i}(t)\right\rangle \leq\left|x_{i}(t)\right|_{X_{0}}\left(\left|x_{j}(t)\right|_{X_{0}}-\left|x_{i}(t)\right|_{X_{0}}\right) \leq 0 .
$$

From $\left\langle x_{i}(t)-P_{X_{i}}\left(x_{i}(t)\right), P_{X_{i}}^{h}\left(x_{i}(t)\right)-P_{X_{i}}\left(x_{i}(t)\right)\right\rangle=0$, we have

$$
\begin{aligned}
& \left\langle x_{i}(t)-P_{X_{0}}\left(x_{i}(t)\right), P_{X_{i}}^{h}\left(x_{i}(t)\right)-P_{X_{i}}\left(x_{i}(t)\right)\right\rangle \\
& =\left\langle P_{X_{i}}\left(x_{i}(t)\right)-P_{X_{0}}\left(x_{i}(t)\right), P_{X_{i}}^{h}\left(x_{i}(t)\right)-P_{X_{i}}\left(x_{i}(t)\right)\right\rangle \\
& \leq\left|x_{i}(t)\right|_{X_{0}} \tan \theta_{i, t}\left|x_{i}(t)\right|_{X_{i}} \\
& \leq \tan \theta_{i, t}\left|x_{i}(t)\right|_{X_{0}}^{2},
\end{aligned}
$$

where the inequalities follow from Lemma 2.1 (ii) by setting $K=X_{i}, y=x_{i}(t), z=$ $P_{X_{0}}\left(x_{i}(t)\right) \in X_{i}$, and $\left|x_{i}(t)\right|_{X_{i}} \leq\left|x_{i}(t)\right|_{X_{0}}$ (due to $X_{0} \subseteq X_{i}$ ). Moreover, it follows from Lemma 2.1 (i) that $\left\langle P_{X_{i}}\left(x_{i}(t)\right)-P_{X_{0}}\left(x_{i}(t)\right), P_{X_{i}}\left(x_{i}(t)\right)-x_{i}(t)\right\rangle \leq 0$ and then

$$
\begin{aligned}
& \left\langle x_{i}(t)-P_{X_{0}}\left(x_{i}(t)\right), P_{X_{i}}\left(x_{i}(t)\right)-x_{i}(t)\right\rangle \\
& =-\left|x_{i}(t)\right|_{X_{i}}^{2}+\left\langle P_{X_{i}}\left(x_{i}(t)\right)-P_{X_{0}}\left(x_{i}(t)\right), P_{X_{i}}\left(x_{i}(t)\right)-x_{i}(t)\right\rangle \\
& \leq-\left|x_{i}(t)\right|_{X_{i}}^{2} .
\end{aligned}
$$


Therefore, based on (17) and (18) we have

$$
\begin{aligned}
& \left\langle x_{i}(t)-P_{X_{0}}\left(x_{i}(t)\right), P_{X_{i}}^{h}\left(x_{i}(t)\right)-x_{i}(t)\right\rangle \\
& =\left\langle x_{i}(t)-P_{X_{0}}\left(x_{i}(t)\right), P_{X_{i}}\left(x_{i}(t)\right)-x_{i}(t)\right\rangle+\left\langle x_{i}(t)-P_{X_{0}}\left(x_{i}(t)\right), P_{X_{i}}^{h}\left(x_{i}(t)\right)-P_{X_{i}}\left(x_{i}(t)\right)\right\rangle \\
& \leq-\left|x_{i}(t)\right|_{X_{i}}^{2}+\tan \theta_{i, t}\left|x_{i}(t)\right|_{X_{0}}^{2} \\
& \leq \tan \theta_{i, t}\left|x_{i}(t)\right|_{X_{0}}^{2} .
\end{aligned}
$$

From (15), (16) and (19), $D^{+} h(t) \leq 2 \alpha_{i, t} \tan \theta_{i, t} h(t) \leq 2 \alpha_{t}^{+} \tan \theta_{t}^{+} h(t)$. Thus, the conclusion follows.

Lemma 5.2 If $\cap_{i=1}^{n} X_{i} \neq \emptyset$ and $\int_{0}^{\infty} \alpha_{t}^{+} \tan \theta_{t}^{+} d t<\infty$, then $\lim _{t \rightarrow \infty} h(t)$ is a finite number.

Proof. Lemma 5.1 implies $h(t) \leq e^{2 \int_{0}^{\infty} \alpha_{s}^{+} \tan \theta_{s}^{+} d s} h(0)$ and then $\bar{h}:=\sup _{t \geq 0} h(t)$ is a finite number. We then show the conclusion by contradiction. Hence suppose there are two limit points $\bar{h}_{1} \neq \bar{h}_{2}$ of $\{h(t)\}_{t \geq 0}$ such that $\lim _{k \rightarrow \infty} h\left(s_{k}^{1}\right)=\bar{h}_{1}$ and $\lim _{k \rightarrow \infty} h\left(s_{k}^{2}\right)=$ $\bar{h}_{2}$. Without loss of generality, we assume $\bar{h}_{1}<\bar{h}_{2}$. Clearly, for any $\varepsilon>0$ for which $(1+\varepsilon)\left(\bar{h}_{1}+\varepsilon\right) \leq \frac{\bar{h}_{1}+\bar{h}_{2}}{2}$, there is an integer $T_{0}>0$ such that $e^{2 \int_{s_{k}^{1}}^{\infty} \alpha_{s}^{+} \tan \theta_{s}^{+} d s} \leq 1+\varepsilon$ and $\left|h\left(s_{k}^{1}\right)-\bar{h}_{1}\right| \leq \varepsilon$ for $k \geq T_{0}$. According to Lemma 5.1, for any $t \geq s_{k}^{1}$ with $k \geq T_{0}$,

$$
h(t) \leq e^{2 \int_{s}^{\infty} \alpha_{s}^{+} \tan \theta_{s}^{+} d s} h\left(s_{k}^{1}\right) \leq(1+\varepsilon)\left(\bar{h}_{1}+\varepsilon\right) \leq \frac{\bar{h}_{1}+\bar{h}_{2}}{2}<\bar{h}_{2},
$$

which contradicts that $\bar{h}_{2}$ is also a limit point of $\{h(t)\}_{t \geq 0}$. Thus, the conclusion follows.

By Lemma 5.2, if $\int_{0}^{\infty} \alpha_{t}^{+} \tan \theta_{t}^{+} d t<\infty$, then the sequence $\{h(t)\}_{t \geq 0}$ converges to a finite number denoted as $h^{*}$,

$$
\lim _{t \rightarrow \infty} h(t)=h^{*} .
$$

Denote $h_{i}^{+}=\limsup _{t \rightarrow \infty} h_{i}(t), h_{i}^{-}=\liminf _{t \rightarrow \infty} h_{i}(t), i \in \mathcal{V}$. Clearly, $0 \leq h_{i}^{-} \leq h_{i}^{+} \leq h^{*}$ for all $i$.

Lemma 5.3 Suppose A1 holds and $\cap_{i=1}^{n} X_{i} \neq \emptyset$. If $\int_{0}^{\infty} \alpha_{t}^{+} \tan \theta_{t}^{+} d t<\infty$ and there exists some node $i_{0} \in \mathcal{V}$ such that $h_{i_{0}}^{-}<h^{*}$, then $h^{*}=0$.

The proof of Lemma 5.3 can be completed with similar arguments in Lemma 4.3 in [18], which is omitted here.

Now it is time to prove Theorem 5.1.

Proof of Theorem 5.1. Denote $\alpha_{t}^{+}=\max _{1 \leq i \leq n} \alpha_{i, t}$. From (13), we find that $\int_{0}^{\infty} \alpha_{t} d t=$ $\infty, \int_{0}^{\infty} \alpha_{t} \tan \theta_{t}^{+} d t<\infty$ are equivalent to $\int_{0}^{\infty} \alpha_{t}^{+} d t=\infty, \int_{0}^{\infty} \alpha_{t}^{+} \tan \theta_{t}^{+} d t<\infty$, respectively.

Based on the similar arguments in Lemmas 5.1 and 5.2, we can show that, for any

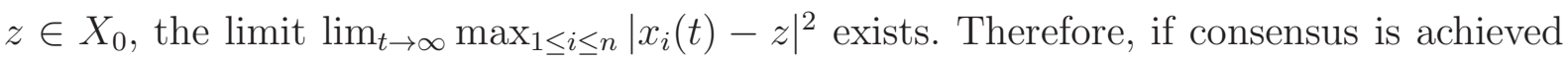


and $h^{*}=0$, all agents will converge to a common point in $X_{0}$. Thus, it suffices to show that consensus is achieved and $h^{*}=0$.

Because

$$
\left|P_{X_{i}}^{h}\left(x_{i}(t)\right)-x_{i}(t)\right|=\frac{\left|x_{i}(t)\right|_{X_{i}}}{\cos \theta_{i, t}} \leq \frac{\sqrt{2 h(t)}}{\cos \theta^{*}}
$$

it follows that, if $h^{*}=0$, the second term on the right-hand side of (6) tends to zero as $t \rightarrow \infty$ and then the consensus is achieved for system (6) by Lemma 2.6. Therefore, it suffices to show $h^{*}=0$ in what follows.

In fact, if there is some node $i_{0}$ with $h_{i_{0}}^{-}<h^{*}$, then $h^{*}=0$ from the previous statements. Therefore, we need to prove $h^{*}=0$ from $h_{i}^{+}=h_{i}^{-}=h^{*}, \forall i$ by contradiction. Clearly, for any $\varepsilon>0$, there is $\bar{t}>0$ such that when $t \geq \bar{t},\left|x_{i}(t)\right|_{X_{0}} \leq \sqrt{2 h^{*}}+\varepsilon=: \phi$. We complete the proof by the following two steps.

Step (i). Suppose $h_{i}^{+}=h_{i}^{-}=h^{*}>0, \forall i$. We claim that consensus can be achieved for system (6).

We first show that $\lim _{t \rightarrow \infty} \alpha_{i, t}\left|x_{i}(t)\right|_{X_{i}}^{2}=0$ by contradiction. Hence suppose there exist $i_{0}$ and an increasing subsequence $\left\{s_{k}\right\}_{k \geq 0}$ with $\lim _{k \rightarrow \infty} s_{k}=\infty$ such that $\alpha_{i_{0}, s_{k}}\left|x_{i_{0}}\left(s_{k}\right)\right|_{X_{i_{0}}}^{2} \geq$ $c$ for some $c>0$. Without loss of generality, we assume $s_{0}$ is sufficiently large such that $s_{0} \geq \bar{t}$ and $\int_{s_{0}}^{\infty} \alpha_{t}^{+} \tan \theta_{t}^{+} d t \leq \varepsilon / \sqrt{2 h^{*}}$.

From Lemma 2.3, the boundedness of system states and (20), we know that $\left|x_{i_{0}}(t)\right|_{X_{i_{0}}}^{2}$ is uniformly continuous on $[0, \infty)$. This along with the uniform continuity of $\alpha_{t}$ again implies that $\alpha_{t}\left|x_{i_{0}}(t)\right|_{X_{i_{0}}}^{2}$ is also uniformly continuous on $[0, \infty)$. Therefore, there is $\delta>0$ such that $\alpha_{i_{0}, t}\left|x_{i_{0}}(t)\right|_{X_{i_{0}}}^{2} \geq c / 2$ when $s_{k} \leq t \leq s_{k}+\delta$. Without loss of generality, we assume $\left[s_{k}, s_{k}+\delta\right] \cap \Delta=\emptyset$ for all $k$. We have

$$
\frac{d h_{i_{0}}(t)}{d t} \leq \sum_{j \in \mathcal{N}_{i_{0}}(t)}\left|x_{i_{0}}(t)\right|_{X_{0}}\left(\left|x_{j}(t)\right|_{X_{0}}-\left|x_{i_{0}}(t)\right|_{X_{0}}\right)-\alpha_{i_{0}, t}\left|x_{i_{0}}(t)\right|_{X_{i_{0}}}^{2}+\alpha_{i_{0}, t} \tan \theta_{i_{0}, t}\left|x_{i_{0}}(t)\right|_{X_{0}}^{2}
$$

and then for $s_{k} \leq t \leq s_{k}+\delta$,

$$
\begin{aligned}
D^{+}\left|x_{i_{0}}(t)\right|_{X_{0}} & \leq \sum_{j \in \mathcal{N}_{i_{0}}(t)}\left(\left|x_{j}(t)\right|_{X_{0}}-\left|x_{i_{0}}(t)\right|_{X_{0}}\right)-\frac{\alpha_{i_{0}, t}\left|x_{i_{0}}(t)\right|_{X_{i_{0}}}^{2}}{\phi}+\alpha_{i_{0}, t} \tan \theta_{t}^{+} \phi \\
& \leq(n-1)\left(\phi-\left|x_{i_{0}}(t)\right|_{X_{0}}\right)-\frac{c}{2 \phi}+\alpha_{t}^{+} \tan \theta_{t}^{+} \phi
\end{aligned}
$$

which leads to

$$
\begin{aligned}
\left|x_{i_{0}}(t)\right|_{X_{0}} & \leq e^{-(n-1)\left(t-s_{k}\right)}\left|x_{i_{0}}\left(s_{k}\right)\right|_{X_{0}}+\left(1-e^{-(n-1)\left(t-s_{k}\right)}\right)\left(\phi-\frac{c}{2(n-1) \phi}\right) \\
& +\phi \int_{s_{k}}^{t} e^{-(n-1)(t-s)} \alpha_{s}^{+} \tan \theta_{s}^{+} d s
\end{aligned}
$$


and then

$$
\left|x_{i_{0}}\left(s_{k}+\delta\right)\right|_{X_{0}} \leq \zeta\left(\sqrt{2 h^{*}}+\varepsilon\right)+(1-\zeta)\left(\sqrt{2 h^{*}}+\varepsilon-\frac{c}{2(n-1) \phi}\right)+\varepsilon \frac{\sqrt{2 h^{*}}+\varepsilon}{\sqrt{2 h^{*}}},
$$

where $0<\zeta=e^{-(n-1) \delta}<1$. We can find that the right-hand side of (22) is less than $\sqrt{2 h^{*}}-\frac{c(1-\zeta)}{4(n-1) \sqrt{2 h^{*}}}$ when $\varepsilon$ is sufficiently small, which contradicts $\lim _{t \rightarrow \infty} h_{i_{0}}(t)=h^{*}$. Thus, $\lim _{t \rightarrow \infty} \alpha_{i, t}\left|x_{i}(t)\right|_{X_{i}}^{2}=0, \forall i$. From Theorem 4.2 we have $0 \leq \alpha_{i, t} \leq C_{i} \alpha^{*}$, and hence $\lim _{t \rightarrow \infty} \alpha_{i, t}\left|x_{i}(t)\right|_{X_{i}}=0, \forall i$. According to the equality in (20) and Lemma 2.6, consensus is achieved for system (6).

Step (ii). Suppose $h_{i}^{+}=h_{i}^{-}=h^{*}>0, \forall i$. We will show that $\liminf _{t \rightarrow \infty} \sum_{i=1}^{n}\left|x_{i}(t)\right|_{X_{i}}^{2}=$ 0 by contradiction.

Hence suppose there is $b>0$ such that $\sum_{i=1}^{n}\left|x_{i}(t)\right|_{X_{i}}^{2} \geq b$ for all sufficiently large $t$. Let $|x(t)|_{X_{0}}=\left(\left|x_{1}(t)\right|_{X_{0}}, \ldots,\left|x_{n}(t)\right|_{X_{0}}\right)^{T}, y(t)=\left(\left|x_{1}(t)\right|_{X_{1}}^{2}, \ldots,\left|x_{n}(t)\right|_{X_{n}}^{2}\right)^{T}$,

$D(t)=\operatorname{diag}\left\{\alpha_{1, t}, \ldots, \alpha_{n, t}\right\}$ (a diagonal matrix with diagonal entries $\alpha_{i, t}$ ). Then by (21) we have

$$
D^{+}|x(t)|_{X_{0}} \leq-\mathcal{L}_{\sigma(t)}|x(t)|_{X_{0}}-\frac{1}{\phi} D(t) y(t)+\phi \alpha_{t}^{+} \tan \theta_{t}^{+} \mathbf{1},
$$

where $\mathcal{L}_{\sigma(t)}$ is the Laplacian of graph $\mathcal{G}_{\sigma(t)}$ with $\left(\mathcal{L}_{\sigma(t)}\right)_{i j}=-1$ if $j \in \mathcal{N}_{i}(t),\left(\mathcal{L}_{\sigma(t)}\right)_{i j}=0$ if $j \neq i, j \notin \mathcal{N}_{i}(t)$ and $\left(\mathcal{L}_{\sigma(t)}\right)_{i i}=\left|\mathcal{N}_{i}(t)\right|$. Recall that $t_{k}, k \geq 0$ are all the switching moments of switching graph $\mathcal{G}_{\sigma}$ with $t_{k+1}-t_{k} \geq \tau, \forall k$. It is easy to see that we can add some new "switching moments" in $\left\{t_{k}\right\}_{k \geq 0}$, denoted as $\left\{t_{k}^{\prime}\right\}_{k \geq 0}$, such that $2 \tau \geq t_{k+1}^{\prime}-t_{k}^{\prime} \geq \tau, \forall k$. From (23) we have

$$
\begin{aligned}
\left|x\left(t_{k+1}^{\prime}\right)\right|_{X_{0}} \leq e^{-\mathcal{L}_{\sigma\left(t_{k}^{\prime}\right)}\left(t_{k+1}^{\prime}-t_{k}^{\prime}\right)}\left|x\left(t_{k}^{\prime}\right)\right|_{X_{0}}+ \\
\quad \int_{t_{k}^{\prime}}^{t_{k+1}^{\prime}} e^{-\mathcal{L}_{\sigma\left(t_{k}^{\prime}\right)}\left(t_{k+1}^{\prime}-t\right)}\left(-\frac{D(t) y(t)}{\phi}+\phi \alpha_{t}^{+} \tan \theta_{t}^{+} \mathbf{1}\right) d t .
\end{aligned}
$$

Note that for any $s>0, e^{-s \mathcal{L}_{\sigma\left(t_{k}^{\prime}\right)}}$ is a stochastic matrix (with nonnegative entries and all row sums are ones) and the graph $\mathcal{G}_{\sigma\left(t_{k}^{\prime}\right)}$ is a subgraph of the graph associated with matrix $e^{-s \mathcal{L}_{\sigma\left(t_{k}^{\prime}\right)}}$. Then applying the similar arguments given in the proof of Theorem 4.1 in [15] we can show that $\liminf _{t \rightarrow \infty} \sum_{i=1}^{n}\left|x_{i}(t)\right|_{X_{i}}^{2}=0$.

Then there is a subsequence $\left\{s_{k}\right\}_{k \geq 0}$ with $\lim _{k \rightarrow \infty} s_{k}=\infty$ such that $\lim _{k \rightarrow \infty}\left|x_{i}\left(s_{k}\right)\right|_{X_{i}}=$ 0 for all $i$. Because we have shown that consensus is achieved in Step (i), $\lim _{k \rightarrow \infty}\left|x_{i}\left(s_{k}\right)\right|_{X_{j}}=$ 0 for all $i, j$, which leads to $\lim _{k \rightarrow \infty} h_{i}\left(s_{k}\right)=0$ for all $i$. Thus, $h^{*}=\lim _{t \rightarrow \infty} h_{i}(t)=0$, which contradicts $h_{i}^{+}=h_{i}^{-}=h^{*}>0$. It follows that $h_{i}^{+}=h_{i}^{-}=h^{*}=0$ and then the first conclusion is proved.

Notice that $\alpha_{i, t}=\alpha_{j, t}=\alpha_{t} \forall i, j, t$ and $P_{X_{i}}^{h}\left(x_{i}(t)\right)=P_{X_{i}}^{a}\left(x_{i}(t)\right)=P_{X_{i}}\left(x_{i}(t)\right)$ in the case of $\theta_{i, t}=0 \forall i$, t. Let $\varpi_{i}(t)=\alpha_{t}\left(P_{X_{i}}\left(x_{i}(t)\right)-x_{i}(t)\right)$. Then $(6)$ can be written as

$$
\dot{x}(t)=-\left(\mathcal{L}_{\sigma(t)} \otimes I_{m}\right) x(t)+\varpi(t),
$$


where $x(t)=\left(x_{1}^{T}(t), \ldots, x_{n}^{T}(t)\right)^{T}$ and $\varpi(t)=\left(\varpi_{1}^{T}(t), \ldots, \varpi_{n}^{T}(t)\right)^{T}$. Hence,

$$
x\left(t_{k+1}^{\prime}\right)=e^{-\left(\mathcal{L}_{\sigma\left(t_{k}^{\prime}\right)} \otimes I_{m}\right)\left(t_{k+1}^{\prime}-t_{k}^{\prime}\right)} x\left(t_{k}^{\prime}\right)+\varphi\left(t_{k}^{\prime}\right),
$$

where $\varphi\left(t_{k}^{\prime}\right)=\int_{t_{k}^{\prime}}^{t_{k+1}^{\prime}} e^{-\left(\mathcal{L}_{\sigma\left(t_{k}^{\prime}\right)} \otimes I_{m}\right)\left(t_{k+1}^{\prime}-t\right)} \varpi(t) d t$. Clearly, $\left|\varpi_{i}(t)\right| \leq \sqrt{2 \bar{h}} \alpha_{t}$, and then $\left|\varphi\left(t_{k}^{\prime}\right)\right| \leq$ $\sqrt{2 n \bar{h}} \int_{t_{k}^{\prime}}^{t_{k+1}^{\prime}} \alpha_{t} d t$. Similar to the arguments given in Theorem 4.2 in [15], we can show the second conclusion.

\section{Empty Intersection Case}

In this section, we discuss the convergence in the empty intersection case (i.e., $\cap_{i=1}^{n} X_{i}=\emptyset$ ) and then show some properties of the optimal solution set in the following two subsections.

\subsection{Convergence Analysis}

The following is the convergence result for the case when $\cap_{i=1}^{n} X_{i}=\emptyset$.

Theorem 6.1 Suppose A1-A3 hold, $\mathcal{G}_{\sigma(t)}, t \geq 0$ are undirected and $\cap_{i=1}^{n} X_{i}=\emptyset$. Then $S D O P$ is solved by system (6) if $\int_{0}^{\infty} \alpha_{t} d t=\infty, \int_{0}^{\infty} \alpha_{t}^{2} d t<\infty$ and $\int_{0}^{\infty} \alpha_{t} \tan \theta_{t}^{+} d t<\infty$; Furthermore, if $\theta_{i, t}=0 \forall i, t$, then it is necessary that $\lim _{t \rightarrow \infty} \alpha_{t}=0$ for (6) to solve $S D O P$.

Remark 6.1 In the case of the exact projection (i.e., $\theta_{i, t} \equiv 0$ ), the stepsize conditions in Theorem 6.1 become $\int_{0}^{\infty} \alpha_{t} d t=\infty$ and $\int_{0}^{\infty} \alpha_{t}^{2} d t<\infty$, which is a continuous-time version of the discrete-time stochastic approximation stepsize condition $\sum_{k=0}^{\infty} \alpha_{k}=\infty$ and $\sum_{k=0}^{\infty} \alpha_{k}^{2}<\infty$ given in [20] to solve the optimization problem $\min \sum_{i=1}^{n} f_{i}$. Therefore, the result in Theorem 6.1 is consistent with those in [20]. Note that [22] proposed distributed continuous-time algorithms for the empty intersection case when the graphs kept connected, which is more restrictive than the UJSC given in this paper.

From Theorems 5.1 and 6.1, we find that the sufficient optimal consensus conditions are essentially different in these two cases. In addition to the conditions in the nonempty intersection case, the square integrability condition is usually required in the empty intersection case.

Before presenting the proof of Theorem 6.1, we show two lemmas. The first one is taken from Lemma 7 in [20].

Lemma 6.1 Let $0<\lambda<1$ and $\left\{b_{k}\right\}_{k \geq 1}$ be a positive sequence. If $\sum_{k=1}^{\infty} b_{k}<\infty$, then $\sum_{k=1}^{\infty} \sum_{r=1}^{k} \lambda^{k-r} b_{r}<\infty$. 
Lemma 6.2 Under A1, if $\int_{0}^{\infty} \alpha_{t}^{2} d t<\infty$ and $\int_{0}^{\infty} \alpha_{t} \tan \theta_{t}^{+} d t<\infty$, then

$$
\int_{0}^{\infty} \alpha_{t}\left|x_{i}(t)-\bar{x}(t)\right| d t<\infty
$$

for all $i$, where $\bar{x}(t)=\frac{1}{n} \sum_{i=1}^{n} x_{i}(t)$.

Proof. Let $H(t)=\max _{1 \leq i, j \leq n}\left|x_{i}(t)-x_{j}(t)\right|$. Since $\left|x_{i}(t)-\bar{x}(t)\right| \leq H(t)$, it suffices to show $\int_{0}^{\infty} \alpha_{t} H(t) d t<\infty$. By Theorem 4.1 (i), (5) and Lemma 2.5, for any $k \geq 0$ and $t$, $k B_{0} \leq t<(k+1) B_{0}$, it holds that

$$
\begin{aligned}
H\left((k+1) B_{0}\right) & \leq \beta H\left(k B_{0}\right)+B_{2} \nu\left(k B_{0}\right), \\
H(t) & \leq H\left(k B_{0}\right)+B_{2} \nu\left(k B_{0}\right),
\end{aligned}
$$

for some $B_{2}>0$, where $\nu\left(k B_{0}\right)=\int_{k B_{0}}^{(k+1) B_{0}} \alpha_{t} d t$. Thus, with (25) we have

$$
\begin{aligned}
\int_{0}^{\infty} \alpha_{t} H(t) d t & =\sum_{k=0}^{\infty} \int_{k B_{0}}^{(k+1) B_{0}} \alpha_{t} H(t) d t \\
& \leq \sum_{k=0}^{\infty} \int_{k B_{0}}^{(k+1) B_{0}} \alpha_{t}\left(H\left(k B_{0}\right)+B_{2} \nu\left(k B_{0}\right)\right) d t \\
& =\sum_{k=0}^{\infty} \nu\left(k B_{0}\right)\left(H\left(k B_{0}\right)+B_{2} \nu\left(k B_{0}\right)\right) .
\end{aligned}
$$

Now we estimate the term in (26). First, by Cauchy-Schwarz inequality $\int g_{1} g_{2} \leq$ $\sqrt{\int g_{1}^{2} \int g_{2}^{2}}$, we have

$$
\sum_{k=0}^{\infty} \nu^{2}\left(k B_{0}\right) \leq B_{0} \sum_{k=0}^{\infty} \int_{k B_{0}}^{(k+1) B_{0}} \alpha_{t}^{2} d t=B_{0} \int_{0}^{\infty} \alpha_{t}^{2} d t<\infty .
$$

Second, by (24) we have $H\left(k B_{0}\right) \leq \beta^{k} H(0)+B_{2} \sum_{r=1}^{k} \beta^{k-r} \nu\left((r-1) B_{0}\right), \forall k \geq 1$. Thus,

$$
\begin{aligned}
\sum_{k=1}^{\infty} H\left(k B_{0}\right) \nu\left(k B_{0}\right) & \leq H(0) \sum_{k=1}^{\infty} \beta^{k} \nu\left(k B_{0}\right)+B_{2} \sum_{k=1}^{\infty} \nu\left(k B_{0}\right) \sum_{r=1}^{k} \beta^{k-r} \nu\left((r-1) B_{0}\right) \\
& \leq \frac{H(0) \beta \alpha^{*} B_{0}}{1-\beta}+\frac{B_{2}}{2} \sum_{k=1}^{\infty} \sum_{r=1}^{k} \beta^{k-r}\left(\nu^{2}\left(k B_{0}\right)+\nu^{2}\left((r-1) B_{0}\right)\right) \\
& \leq \frac{H(0) \beta \alpha^{*} B_{0}}{1-\beta}+\frac{B_{2}}{2(1-\beta)} \sum_{k=1}^{\infty} \nu^{2}\left(k B_{0}\right)+\frac{B_{2}}{2} \sum_{k=1}^{\infty} \sum_{r=1}^{k} \beta^{k-r} \nu^{2}\left((r-1) B_{0}\right) \\
& <\infty,
\end{aligned}
$$

where the second inequality follows from $\nu\left(k B_{0}\right) \leq \alpha^{*} B_{0}$, the third one from $\sum_{r=1}^{k} \beta^{k-r} \leq$ $\frac{1}{1-\beta}, \forall k$, and the last one from (27) and Lemma 6.1. Thus, the conclusion follows from (26), (27) and (28). 
It is time to give the proof of Theorem 6.1.

Proof of Theorem 6.1. We rewrite (6) as

$$
\dot{x}_{i}(t)=\sum_{j \in \mathcal{N}_{i}(t)}\left(x_{j}(t)-x_{i}(t)\right)+\alpha_{t}\left(P_{X_{i}}^{h}\left(x_{i}(t)\right)-x_{i}(t)\right)+\phi_{i}(t)
$$

where $\phi_{i}(t)=\left(\alpha_{i, t}-\alpha_{t}\right)\left(P_{X_{i}}^{h}\left(x_{i}(t)\right)-x_{i}(t)\right)$. From the definition of $P_{X_{i}}^{h}$, we have $\mid P_{X_{i}}^{h}\left(x_{i}(t)\right)-$ $x_{i}(t) \mid=\frac{\left|x_{i}(t)\right|_{X_{i}}}{\cos \theta_{i, t}} \leq \frac{\eta}{\cos \theta_{i, t}}$, where

$$
\eta=\sup _{i, j, t}\left\{\left|x_{i}(t)-x^{*}\right|,|\bar{x}(t)|_{X_{i}},\left|x_{i}(t)\right|_{X_{j}}\right\}
$$

is a finite number by Theorem 4.1. Moreover, it follows from (13) that $\left|\alpha_{i, t}-\alpha_{t}\right| \leq$ $\left(C_{i, t}-1\right) \alpha_{t} \leq \frac{1}{\sin \mu_{i}} \alpha_{t} \sin \theta_{i, t}$. Then from the preceding two estimates,

$$
\left|\phi_{i}(t)\right| \leq \frac{\eta}{\sin \mu_{i}} \alpha_{t} \tan \theta_{i, t}
$$

Take $x^{*} \in \arg \min \sum_{i=1}^{n}|x|_{X_{i}}^{2}$. Let $t \notin \Delta$. Clearly,

$$
\begin{aligned}
\frac{d\left|x_{i}(t)-x^{*}\right|^{2}}{d t} & =2\left\langle x_{i}(t)-x^{*}, \dot{x}_{i}(t)\right\rangle \\
& =2\left\langle x_{i}(t)-x^{*}, \sum_{j \in \mathcal{N}_{i}(t)}\left(x_{j}(t)-x_{i}(t)\right)+\alpha_{t}\left(P_{X_{i}}^{h}\left(x_{i}(t)\right)-x_{i}(t)\right)+\phi_{i}(t)\right\rangle .
\end{aligned}
$$

Because $\mathcal{G}_{\sigma(t)}$ is undirected,

$$
\begin{aligned}
& \frac{d \sum_{i=1}^{n}\left|x_{i}(t)-x^{*}\right|^{2}}{d t} \\
& =-2 \sum_{j \in \mathcal{N}_{i}(t)}\left|x_{j}(t)-x_{i}(t)\right|^{2}+2 \sum_{i=1}^{n}\left\langle x_{i}(t)-x^{*}, \alpha_{t}\left(P_{X_{i}}^{h}\left(x_{i}(t)\right)-x_{i}(t)\right)+\phi_{i}(t)\right\rangle \\
& \leq 2 \alpha_{t} \sum_{i=1}^{n}\left\langle x_{i}(t)-x^{*}, P_{X_{i}}^{h}\left(x_{i}(t)\right)-x_{i}(t)\right\rangle+2 \sum_{i=1}^{n}\left\langle x_{i}(t)-x^{*}, \phi_{i}(t)\right\rangle .
\end{aligned}
$$

Then we estimate the first term in (30). Note that

$$
\left\langle x_{i}(t)-x^{*}, P_{X_{i}}^{h}\left(x_{i}(t)\right)-P_{X_{i}}\left(x_{i}(t)\right)\right\rangle \leq\left|x_{i}(t)-x^{*}\right| \tan \theta_{i, t}\left|x_{i}(t)\right|_{X_{i}} \leq \eta^{2} \tan \theta_{t}^{+} .
$$

We also have

$$
\sum_{i=1}^{n}\left\langle x_{i}(t)-x^{*}, P_{X_{i}}\left(x_{i}(t)\right)-x_{i}(t)\right\rangle=-\left\langle\bar{x}(t)-x^{*}, \sum_{i=1}^{n}\left(\bar{x}(t)-P_{X_{i}}(\bar{x}(t))\right)\right\rangle+\varrho(t),
$$

where

$\varrho(t)=\sum_{i=1}^{n}\left\langle x_{i}(t)-\bar{x}(t), P_{X_{i}}(\bar{x}(t))-\bar{x}(t)\right\rangle+\sum_{i=1}^{n}\left\langle x_{i}(t)-x^{*}, P_{X_{i}}\left(x_{i}(t)\right)-P_{X_{i}}(\bar{x}(t))+\bar{x}(t)-x_{i}(t)\right\rangle$. 
Clearly, the first term in $\varrho(t)$ is not greater than $\eta \sum_{i=1}^{n}\left|x_{i}(t)-\bar{x}(t)\right|$ and the second term in $\varrho(t)$ is not greater than $2 \eta \sum_{i=1}^{n}\left|x_{i}(t)-\bar{x}(t)\right|$ by Lemma 2.1 (iv). Moreover, by (29), the second term in (30) is not greater than $2 n \eta^{2} \alpha_{t} \tan \theta_{t}^{+} / \sin \mu_{-}$, where $\mu_{-}=\min _{1 \leq i \leq n} \mu_{i}$. Denote $\psi(t)=\left\langle\bar{x}(t)-x^{*}, \sum_{i=1}^{n}\left(\bar{x}(t)-P_{X_{i}}(\bar{x}(t))\right)\right\rangle, \varsigma(t)=6 \eta\left(\sum_{i=1}^{n} \alpha_{t}\left|x_{i}(t)-\bar{x}(t)\right|\right)+$ $2 n \eta^{2}\left(1+\frac{1}{\sin \mu_{-}}\right) \alpha_{t} \tan \theta_{t}^{+}$. In light of (30), (31) and (32), we have

$$
\frac{d \sum_{i=1}^{n}\left|x_{i}(t)-x^{*}\right|^{2}}{d t} \leq-2 \alpha_{t} \psi(t)+\varsigma(t) \leq \varsigma(t)
$$

because $\psi(t)$ is nonnegative, following from (1) and the convexity of objective function $f$, that is,

$$
\psi(t)=\left\langle\bar{x}(t)-x^{*}, \frac{1}{2} \nabla f(\bar{x}(t))\right\rangle \geq \frac{1}{2}\left(f(\bar{x}(t))-f\left(x^{*}\right)\right) \geq 0 .
$$

We next show that $\lim _{t \rightarrow \infty} \sum_{i=1}^{n}\left|x_{i}(t)-x^{*}\right|^{2}$ is a finite number by contradiction. Let us suppose there exist $\left\{s_{k}\right\}_{k \geq 0}$ with $s_{k} \rightarrow \infty$ and $\varepsilon>0$ such that $\sum_{i=1}^{n}\left|x_{i}\left(s_{2 k+1}\right)-x^{*}\right|^{2}-$ $\sum_{i=1}^{n}\left|x_{i}\left(s_{2 k}\right)-x^{*}\right|^{2} \geq \varepsilon$ for all $k$. According to Lemma 6.2, $\int_{0}^{\infty} \sum_{i=1}^{n} \alpha_{t}\left|x_{i}(t)-\bar{x}(t)\right| d t<\infty$. Therefore, there is $K_{0}>0$ such that $\int_{K_{0}}^{\infty} \sum_{i=1}^{n} \alpha_{t}\left|x_{i}(t)-\bar{x}(t)\right| d t \leq \frac{\varepsilon}{24 \eta}$ and $\int_{K_{0}}^{\infty} \alpha_{t} \tan \theta_{t}^{+} d t \leq$ $\frac{\varepsilon}{8 n \eta^{2}\left(1+1 / \sin \mu_{-}\right)}$. By (33), we have that, for sufficiently large $k$ for which $t_{2 k} \geq K_{0}$,

$$
\begin{aligned}
\varepsilon & \leq \sum_{i=1}^{n}\left|x_{i}\left(s_{2 k+1}\right)-x^{*}\right|^{2}-\sum_{i=1}^{n}\left|x_{i}\left(s_{2 k}\right)-x^{*}\right|^{2} \\
& \leq 6 \eta \int_{K_{0}}^{\infty} \alpha_{t}\left(\sum_{i=1}^{n}\left|x_{i}(t)-\bar{x}(t)\right|\right) d t+2 n \eta^{2} \int_{K_{0}}^{\infty} \alpha_{t} \tan \theta_{t}^{+} d t \\
& \leq \frac{\varepsilon}{2}
\end{aligned}
$$

which yields a contradiction. Hence, $\lim _{t \rightarrow \infty} \sum_{i=1}^{n}\left|x_{i}(t)-x^{*}\right|^{2}$ is a finite number.

Thus, it follows from (33) that

$$
2 \int_{0}^{\infty} \alpha_{t}\left\langle\bar{x}(t)-x^{*}, \sum_{i=1}^{n}\left(\bar{x}(t)-P_{X_{i}}(\bar{x}(t))\right)\right\rangle=\int_{0}^{\infty} \alpha_{t}\left\langle\bar{x}(t)-x^{*}, \nabla f(\bar{x}(t))\right\rangle<\infty .
$$

Due to $\int_{0}^{\infty} \alpha_{t} d t=\infty$, there is a subsequence $\left\{t_{r}\right\}_{r \geq 0}$ such that $\lim _{r \rightarrow \infty}\left\langle\bar{x}\left(t_{r}\right)-x^{*}, \nabla f\left(\bar{x}\left(t_{r}\right)\right)\right\rangle=$ 0 . Since the system states are bounded, without loss of generality we assume $\lim _{r \rightarrow \infty} \bar{x}\left(t_{r}\right)=$ $\hat{x}$ for some $\hat{x}$ (otherwise we can further find a subsequence of $\left\{t_{r}\right\}_{r \geq 0}$ ). Since $\nabla f$ is continuous, $\left\langle\hat{x}-x^{*}, \nabla f(\hat{x})\right\rangle=0$, which leads to $f\left(x^{*}\right) \geq f(\hat{x})+\left\langle x^{*}-\hat{x}, \nabla f(\hat{x})\right\rangle=f(\hat{x})$. Thus, $\hat{x} \in \arg \min f$.

Replacing $x^{*}$ with $\hat{x}$, we can similarly show that $\lim _{t \rightarrow \infty} \sum_{i=1}^{n}\left|x_{i}(t)-\hat{x}\right|^{2}$ is also a finite number, denoted as $\rho$. Moreover, the uniform continuity of $\alpha_{t}$ and $\int_{0}^{\infty} \alpha_{t}^{2} d t<\infty$ imply $\lim _{t \rightarrow \infty} \alpha_{t}=0$. Therefore, consensus is achieved by Lemma 2.6 and then $\lim _{r \rightarrow \infty} x_{i}\left(t_{r}\right)=$ 
$\hat{x}$. Hence $\rho=0$, which in return implies $\lim _{t \rightarrow \infty} x_{i}(t)=\hat{x}$ for all $i$. Then the first part is completed.

Now we show the second part. Notice that $\theta_{i, t} \equiv 0$ implies that $\alpha_{i, t}=\alpha_{j, t}=\alpha_{t} \forall i, j, t$ and $P_{X_{i}}^{h}\left(x_{i}(t)\right)=P_{X_{i}}^{a}\left(x_{i}(t)\right)=P_{X_{i}}\left(x_{i}(t)\right)$. Let $x^{*} \in \arg \min \sum_{i=1}^{n}|x|_{X_{i}}^{2}$ be the point with $\lim _{t \rightarrow \infty} x_{i}(t)=x^{*}$ for all $i$. According to the boundedness of system states, the non-expansive property Lemma 2.1 (iv) and the uniform continuity of $\alpha_{t}, \dot{x}_{i}(t)$ is uniformly continuous with respect to time intervals $\left(t_{k}, t_{k+1}\right), k \geq 0$, which, along with $\lim _{t \rightarrow \infty} x_{i}(t)=x^{*}$, leads to $\lim _{t \rightarrow \infty} \dot{x}_{i}(t)=0$ by Lemma 2.7 . Therefore, because the network achieves a consensus,

$$
\lim _{t \rightarrow \infty} \alpha_{t}\left(P_{X_{i}}\left(x_{i}(t)\right)-x_{i}(t)\right)=\lim _{t \rightarrow \infty} \alpha_{t}\left(P_{X_{i}}\left(x^{*}\right)-x^{*}\right)=0, i=1, \ldots, n
$$

According to $\cap_{i=1}^{n} X_{i}=\emptyset, x^{*} \notin X_{i}$ for at least one $i$. Thus, $\lim _{t \rightarrow \infty} \alpha_{t}=0$.

\subsection{Optimal Solutions}

Theorem 6.1 showed that all agents consensually converge to an optimal solution of $\min \sum_{i=1}^{n}|x|_{X_{i}}^{2}$ under certain conditions. Next, we show some properties of the optimal solution set of $\min \sum_{i=1}^{n}|x|_{X_{i}}^{2}$, denoted as $X^{*}$. According to Lemma 2.3, the optimal solution $x^{*} \in X^{*}$ must satisfy

$$
\nabla \sum_{i=1}^{n}\left|x^{*}\right|_{X_{i}}^{2}=2 \sum_{i=1}^{n}\left(x^{*}-P_{X_{i}}\left(x^{*}\right)\right)=0,
$$

or equivalently, $x^{*}=\frac{\sum_{i=1}^{n} P_{X_{i}}\left(x^{*}\right)}{n}$. Then we have the following results.

Before showing some properties of the optimal solutions, we give a lemma first.

Lemma 6.3 Let $K$ be a closed convex set in $\mathbb{R}^{m}$. Then

(i) $\left\langle y-z, P_{K}(y)-P_{K}(z)\right\rangle \geq\left|P_{K}(y)-P_{K}(z)\right|^{2}$ for any $y$ and $z$;

(ii) $\left|P_{K}(y)-P_{K}(z)\right|=|y-z|$ if and only if $y-P_{K}(y)=z-P_{K}(z)$.

Proof. (i) follows from

$$
\begin{aligned}
\left\langle y-z, P_{K}(y)-P_{K}(z)\right\rangle & =\left\langle y-P_{K}(y), P_{K}(y)-P_{K}(z)\right\rangle+\left|P_{K}(y)-P_{K}(z)\right|^{2} \\
& +\left\langle P_{K}(z)-z, P_{K}(y)-P_{K}(z)\right\rangle \\
& \geq\left|P_{K}(y)-P_{K}(z)\right|^{2}
\end{aligned}
$$

because $\left\langle y-P_{K}(y), P_{K}(y)-P_{K}(z)\right\rangle \geq 0$ and $\left\langle P_{K}(z)-z, P_{K}(y)-P_{K}(z)\right\rangle \geq 0$ by Lemma 2.1 (i). 
For (ii), the sufficiency is obvious. The necessity can be obtained from

$$
\begin{aligned}
\left|y-P_{K}(y)-\left(z-P_{K}(z)\right)\right|^{2} & =|y-z|^{2}+\left|P_{K}(z)-P_{K}(y)\right|^{2}+2\left\langle y-z, P_{K}(z)-P_{K}(y)\right\rangle \\
& =2|y-z|^{2}+2\left\langle y-z, P_{K}(z)-P_{K}(y)\right\rangle \\
& \leq 2|y-z|^{2}-2\left|P_{K}(y)-P_{K}(z)\right|^{2} \\
& =0,
\end{aligned}
$$

where the inequality follows from (i) of this lemma.

Let $X^{*}$ be the optimal solution set of $\min \sum_{i=1}^{n}|x|_{X_{i}}^{2}$. Then we have the following results.

Theorem 6.2 (i) For any $x^{*}, y^{*} \in X^{*}$, we have $x^{*}-P_{X_{i}}\left(x^{*}\right)=y^{*}-P_{X_{i}}\left(y^{*}\right), \quad i=1, \ldots, n$;

(ii) For any $i$, either $X^{*} \subseteq X_{i}$ or $X^{*} \cap X_{i}=\emptyset$;

(iii) Let $x^{*} \in X^{*}, x^{*} \notin X_{i}$ for some $i$. Then $X^{*} \cap$ line $\left(x^{*}, P_{X_{i}}\left(x^{*}\right)\right)=\left\{x^{*}\right\}$.

Proof. (i) Since $x^{*}=\frac{\sum_{i=1}^{n} P_{X_{i}}\left(x^{*}\right)}{n}$ and $y^{*}=\frac{\sum_{i=1}^{n} P_{X_{i}}\left(y^{*}\right)}{n}$,

$$
\begin{aligned}
\left|x^{*}-y^{*}\right| & =\left|\frac{\sum_{i=1}^{n}\left(P_{X_{i}}\left(x^{*}\right)-P_{X_{i}}\left(y^{*}\right)\right)}{n}\right| \\
& \leq \frac{\sum_{i=1}^{n}\left|P_{X_{i}}\left(x^{*}\right)-P_{X_{i}}\left(y^{*}\right)\right|}{n} \\
& \leq\left|x^{*}-y^{*}\right|
\end{aligned}
$$

from Lemma 2.1 (iv). Therefore, $\left|P_{X_{i}}\left(x^{*}\right)-P_{X_{i}}\left(y^{*}\right)\right|=\left|x^{*}-y^{*}\right|$ for all $i$, which implies the conclusion by Lemma 6.3 (ii).

(ii) This is straightforward from (i).

(iii) Let $z^{*} \in X^{*} \cap \operatorname{line}\left(x^{*}, P_{X_{i}}\left(x^{*}\right)\right), z^{*} \neq x^{*}$. If $z^{*}$ locates the half-line with $P_{X_{i}}\left(x^{*}\right)$ as the starting point and $x^{*}-P_{X_{i}}\left(x^{*}\right)$ as the direction, then $P_{X_{i}}\left(z^{*}\right)=P_{X_{i}}\left(x^{*}\right)$ by Lemma 2.1 (i) (note that Lemma 2.1 is an equivalent definition of convex projection). Therefore, $x^{*}-P_{X_{i}}\left(x^{*}\right) \neq z^{*}-P_{X_{i}}\left(z^{*}\right)$, which contradicts what we have proven in (i) since both $x^{*}$ and $z^{*}$ are optimal solutions. If $z^{*}$ locates the half-line with $P_{X_{i}}\left(x^{*}\right)$ as the starting point and $P_{X_{i}}\left(x^{*}\right)-x^{*}$ as the direction, then $P_{X_{i}}\left(x^{*}\right)$ is also an optimal solution since the optimal solution set $X^{*}$ is a convex set and $P_{X_{i}}\left(x^{*}\right)$ can be written as a convex combination of $x^{*}, z^{*}$. Then $0=P_{X_{i}}\left(x^{*}\right)-P_{X_{i}}\left(P_{X_{i}}\left(x^{*}\right)\right) \neq x^{*}-P_{X_{i}}\left(x^{*}\right)$, which also yields a contradiction since both $x^{*}$ and $P_{X_{i}}\left(x^{*}\right)$ are optimal solutions. Thus, the conclusion follows.

\section{$7 \quad$ Fixed Stepsize and Approximate Angle}

In this section, we consider the constant stepsize and approximate angle case. The following result is about the convergence error between the agents' estimates and the optimal point in terms of the stepsize and approximate angle. 
Theorem 7.1 Consider system (5) with $\alpha_{t} \equiv \alpha>0$ and $0 \leq \theta_{i, t} \equiv \theta_{i}<\pi / 2$, under A1-A3 and that $\mathcal{G}_{\sigma(t)}$ is undirected for $t \geq 0$.

(i) Suppose $\cap_{i=1}^{n} \operatorname{int}\left(X_{i}\right) \neq \emptyset$ (implies $\cap_{i=1}^{n} X_{i} \neq \emptyset$ ). Then

$$
\begin{aligned}
\limsup _{t \rightarrow \infty}\left|x_{i}(t)\right|_{X_{0}} \leq & \sqrt{\kappa_{S} n \eta d_{0}\left(\frac{4-2 \beta}{1-\beta} B_{0} B_{1} C \sqrt{1+\left(\tan \theta^{+}\right)^{2}} \alpha+C \tan \theta^{+}\right)} \\
& +\frac{2-\beta}{1-\beta} B_{0} B_{1} C \sqrt{1+\left(\tan \theta^{+}\right)^{2}} \eta \alpha .
\end{aligned}
$$

(ii) Suppose $\cap_{i=1}^{n} X_{i}=\emptyset$ and $f(x)=\sum_{i=1}^{n}|x|_{X_{i}}^{2}$ is l-strongly convex. Let $x^{*}$ be the unique optimal solution of $\min f$. Then

$$
\begin{aligned}
\limsup _{t \rightarrow \infty}\left|x_{i}(t)-x^{*}\right| \leq & \sqrt{\frac{4 n \eta^{2}}{\ell}\left(\frac{4-2 \beta}{1-\beta} B_{0} B_{1} C \sqrt{1+\left(\tan \theta^{+}\right)^{2}} \alpha+C \tan \theta^{+}\right)} \\
& +\frac{2-\beta}{1-\beta} B_{0} B_{1} C \sqrt{1+\left(\tan \theta^{+}\right)^{2}} \eta \alpha
\end{aligned}
$$

with $S=X_{c}+\mathbf{B}\left(0, r_{0}\right), C=\max _{1 \leq i \leq n} C_{i}, \theta^{+}=\max _{1 \leq i \leq n} \theta_{i}, C_{i}$ defined in (13), $\kappa_{S}$ defined in Lemma 2.2, and $\beta, B_{0}$ and $B_{1}$ defined in Lemma 2.5, where $d_{0}=\sup _{i, t}\left|x_{i}(t)\right|_{X_{0}}$, which is finite by Theorem 4.1.

Proof. Similar to $(24), H\left((k+1) B_{0}\right) \leq \beta H\left(k B_{0}\right)+B_{0} B_{1} C \sqrt{1+\left(\tan \theta^{+}\right)^{2}} \eta \alpha$, and then

$$
H\left(k B_{0}\right) \leq \beta^{k} H(0)+\left(1+\beta+\cdots+\beta^{k-1}\right) B_{0} B_{1} C \sqrt{1+\left(\tan \theta^{+}\right)^{2}} \eta \alpha,
$$

along with $H(t) \leq H\left(k B_{0}\right)+B_{0} B_{1} C \sqrt{1+\left(\tan \theta^{+}\right)^{2}} \eta \alpha, \forall k B_{0} \leq t<(k+1) B_{0}$, implies

$$
\begin{aligned}
H(t) & \leq \beta^{k} H(0)+\left(2+\beta+\cdots+\beta^{k-1}\right) B_{0} B_{1} C \sqrt{1+\left(\tan \theta^{+}\right)^{2}} \eta \alpha \\
& \leq \frac{H(0)}{\beta^{\frac{1}{B_{0}}}}\left(\beta^{\frac{1}{B_{0}}}\right)^{t}+\frac{2-\beta}{1-\beta} B_{0} B_{1} C \sqrt{1+\left(\tan \theta^{+}\right)^{2}} \eta \alpha
\end{aligned}
$$

when $k B_{0} \leq t<(k+1) B_{0}$. Then

$$
\limsup _{t \rightarrow \infty} H(t) \leq \frac{2-\beta}{1-\beta} B_{0} B_{1} C \sqrt{1+\left(\tan \theta^{+}\right)^{2}} \eta \alpha .
$$

Clearly, (5) can be written as

$$
\dot{x}_{i}(t)=\sum_{j \in \mathcal{N}_{i}(t)}\left(x_{j}(t)-x_{i}(t)\right)+\alpha\left(P_{X_{i}}(\bar{x}(t))-\bar{x}(t)\right)+\omega_{i}(t), i=1, \ldots, n,
$$

where $\bar{x}(t)=\frac{1}{n} \sum_{i=1}^{n} x_{i}(t)$,

$$
\omega_{i}(t)=\alpha\left(P_{X_{i}}\left(x_{i}(t)\right)-P_{X_{i}}(\bar{x}(t))+\bar{x}(t)-x_{i}(t)\right)+\alpha\left(P_{X_{i}}^{a}\left(x_{i}(t)\right)-P_{X_{i}}\left(x_{i}(t)\right)\right) .
$$


The first term in $\omega_{i}(t)$ is not greater than $2 \alpha H(t)$ by Lemma 2.1 (iv) and the inequality $\left|\bar{x}(t)-x_{i}(t)\right| \leq H(t)$, and the second term is not greater than $C \eta \alpha \tan \theta^{+}$due to the relation

$$
\frac{\left|P_{X_{i}}^{a}\left(x_{i}(t)\right)-P_{X_{i}}\left(x_{i}(t)\right)\right|}{\left|P_{X_{i}}^{h}\left(x_{i}(t)\right)-P_{X_{i}}\left(x_{i}(t)\right)\right|}=\frac{\sin \left(\frac{\pi}{2}+\theta_{i, t}\right)}{\sin \mu_{i, t}} \leq \frac{1}{\sin \mu_{i}} \leq C_{i}
$$

where the equality follows from the well-known law of sines:

$$
\frac{a_{1}}{\sin A_{1}}=\frac{a_{2}}{\sin A_{2}}=\frac{a_{3}}{\sin A_{3}}
$$

with $a_{1}, a_{2}, a_{3}$ the lengths of the sides of a triangle, and $A_{1}, A_{2}, A_{3}$ the opposite angles. Hence,

$$
\left|\omega_{i}(t)\right| \leq 2 \alpha H(t)+C \eta \alpha \tan \theta^{+}
$$

Therefore, from (36) and the undirectedness of $\mathcal{G}_{\sigma(t)}$, we have

$$
\dot{\bar{x}}(t)=\frac{1}{n} \sum_{i=1}^{n} \dot{x}_{i}(t)=\frac{\alpha}{n} \sum_{i=1}^{n}\left(P_{X_{i}}(\bar{x}(t))-\bar{x}(t)\right)+\frac{1}{n} \sum_{i=1}^{n} \omega_{i}(t) .
$$

We complete the proof for both the nonempty intersection and empty intersection case.

(i) If $\cap_{i=1}^{n} \operatorname{int}\left(X_{i}\right) \neq \emptyset$, then, from Lemma 2.3 , for any $t \notin \Delta$,

$$
\begin{aligned}
\frac{d|\bar{x}(t)|_{X_{0}}^{2}}{d t} & =\frac{2 \alpha}{n}\left\langle\bar{x}(t)-P_{X_{0}}(\bar{x}(t)), \sum_{i=1}^{n}\left(P_{X_{i}}(\bar{x}(t))-\bar{x}(t)\right)\right\rangle+\frac{2}{n}\left\langle\bar{x}(t)-P_{X_{0}}(\bar{x}(t)), \sum_{i=1}^{n} \omega_{i}(t)\right\rangle \\
& \leq-\frac{2 \alpha}{n} \sum_{i=1}^{n}|\bar{x}(t)|_{X_{i}}^{2}+\left(4 \alpha H(t)+2 C \eta \alpha \tan \theta^{+}\right) d_{0} \\
& \leq-\frac{2 \alpha}{n} \max _{1 \leq i \leq n}|\bar{x}(t)|_{X_{i}}^{2}+\left(4 \alpha H(t)+2 C \eta \alpha \tan \theta^{+}\right) d_{0} \\
& \leq-\frac{2 \alpha}{\kappa_{S} n}|\bar{x}(t)|_{X_{0}}^{2}+\left(4 \alpha H(t)+2 C \eta \alpha \tan \theta^{+}\right) d_{0},
\end{aligned}
$$

where the first inequality follows from (18) (replacing $x_{i}(t)$ with $\bar{x}(t)$ ) and (37); the third one from Lemma 2.2. As a result, we obtain that for any $t \geq t_{0} \geq 0$,

$$
|\bar{x}(t)|_{X_{0}}^{2} \leq e^{-\frac{2 \alpha}{\kappa_{S}{ }^{n}}\left(t-t_{0}\right)}\left|\bar{x}\left(t_{0}\right)\right|_{X_{0}}^{2}+e^{-\frac{2 \alpha}{\kappa_{S} n} t} \int_{t_{0}}^{t} e^{\frac{2 \alpha}{\kappa_{S} n} s}\left(4 \alpha H(s)+2 C \eta \alpha \tan \theta^{+}\right) d_{0} d s,
$$

which combines with (35) imply

$$
\limsup _{t \rightarrow \infty}|\bar{x}(t)|_{X_{0}}^{2} \leq \frac{\kappa_{S} n d_{0}}{2 \alpha}\left(4 \alpha^{2} \frac{2-\beta}{1-\beta} B_{0} B_{1} C \sqrt{1+\left(\tan \theta^{+}\right)^{2}} \eta+2 C \eta \alpha \tan \theta^{+}\right) .
$$

This implies the conclusion by noticing the relation $\left|x_{i}(t)\right|_{X_{0}}-|\bar{x}(t)|_{X_{0}} \leq\left|\bar{x}(t)-x_{i}(t)\right| \leq$ $H(t)$ and (35). 
(ii) If $\cap_{i=1}^{n} X_{i}=\emptyset$ and $f(x)=\sum_{i=1}^{n}|x|_{X_{i}}^{2}$ is $\ell$-strongly convex, then when $t \notin \Delta$,

$$
\begin{aligned}
\frac{d\left|\bar{x}(t)-x^{*}\right|^{2}}{d t} & =\frac{2 \alpha}{n}\left\langle\bar{x}(t)-x^{*}, \sum_{i=1}^{n}\left(P_{X_{i}}(\bar{x}(t))-\bar{x}(t)\right)\right\rangle+\frac{2}{n}\left\langle\bar{x}(t)-x^{*}, \sum_{i=1}^{n} \omega_{i}(t)\right\rangle \\
& \leq-\frac{\alpha}{n}\left(f(\bar{x}(t))-f\left(x^{*}\right)\right)+\left(4 \alpha H(t)+2 C \eta \alpha \tan \theta^{+}\right) \eta \\
& \leq-\frac{\alpha \ell}{2 n}\left|\bar{x}(t)-x^{*}\right|^{2}+\left(4 \alpha H(t)+2 C \eta \alpha \tan \theta^{+}\right) \eta
\end{aligned}
$$

where the first inequality follows from (34) and the second one from (2). Thus, the conclusion can be obtained with a proof similar to that for (i).

\section{Numerical Examples}

In this section, we provide an example to illustrate the above convergence results.

Consider a network of three agents with node set $\mathcal{V}=\{1,2,3\}$. The convex set $X_{i}$ of each agent $i$ is the ball in $\mathbb{R}^{2}$ with center $c_{i}$ and radius $r_{i}$. Let $\alpha_{t}=\frac{20}{t+20}, \theta_{i, t}=\frac{1}{t+50}$, which satisfy the conditions in Theorems 5.1 and 6.1. We next present the state trajectories of the three agents for the nonempty and empty intersection cases from time $t=0$ to $t=2000$, respectively.

(i) Nonempty intersection case with

$$
c_{1}=(-1,0)^{T}, c_{2}=(1,0)^{T}, c_{3}=(0,-2)^{T}, r_{1}=2, r_{2}=1, r_{3}=2
$$

The graphs are periodically switching over the two directed graphs $G_{1}=\left(\mathcal{V}, \mathcal{E}_{1}\right), G_{2}=$ $\left(\mathcal{V}, \mathcal{E}_{2}\right)$ with period 1 , where $\mathcal{E}_{1}=\{(2,1),(3,2)\}, \mathcal{E}_{2}=\{(1,3)\}$. The initial conditions are $x_{1}(0)=(-4,3), x_{2}(0)=(3,5), x_{3}(0)=(-6,-3)$, which are marked as $\circ$ in Fig. 3.

(ii) Empty intersection case with

$$
c_{1}=(-\sqrt{3}, 0)^{T}, c_{2}=(\sqrt{3}, 0)^{T}, c_{3}=(0,-3)^{T}, r_{1}=r_{2}=r_{3}=1 .
$$

In this case, the (unique) optimal solution is $(0,-1)$. The graphs are periodically switching over the two undirected graphs $G_{1}=\left(\mathcal{V}, \mathcal{E}_{1}\right), G_{2}=\left(\mathcal{V}, \mathcal{E}_{2}\right)$ with period 1 , where $\mathcal{E}_{1}=$ $\{(3,2)\}, \mathcal{E}_{2}=\{(1,2)\}$. The initial conditions are $x_{1}(0)=(-3,3), x_{2}(0)=(4,2), x_{3}(0)=$ $(-5,-3)$, which are marked as $\circ$ in Fig. 4 .

\section{Conclusions}

In this paper, a continuous-time method was proposed to cooperatively solve the SDOP by a group of agents with the help of graph theory, convex analysis and geometric technique. 


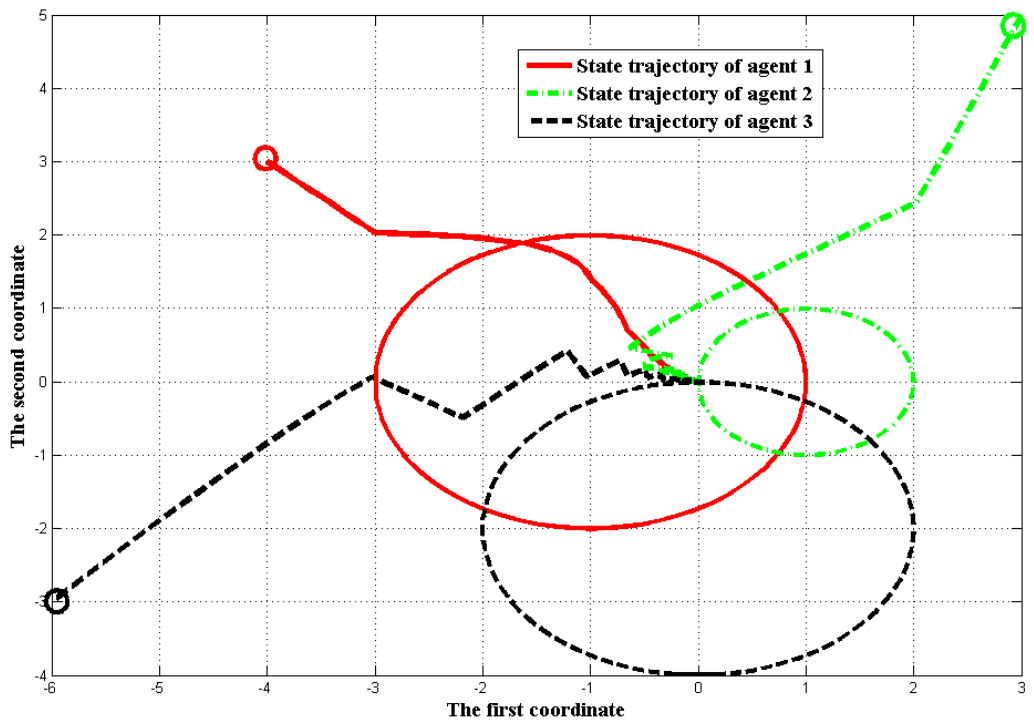

Figure 3: In the nonempty intersection case, all agents converge to a common point in the intersection set.

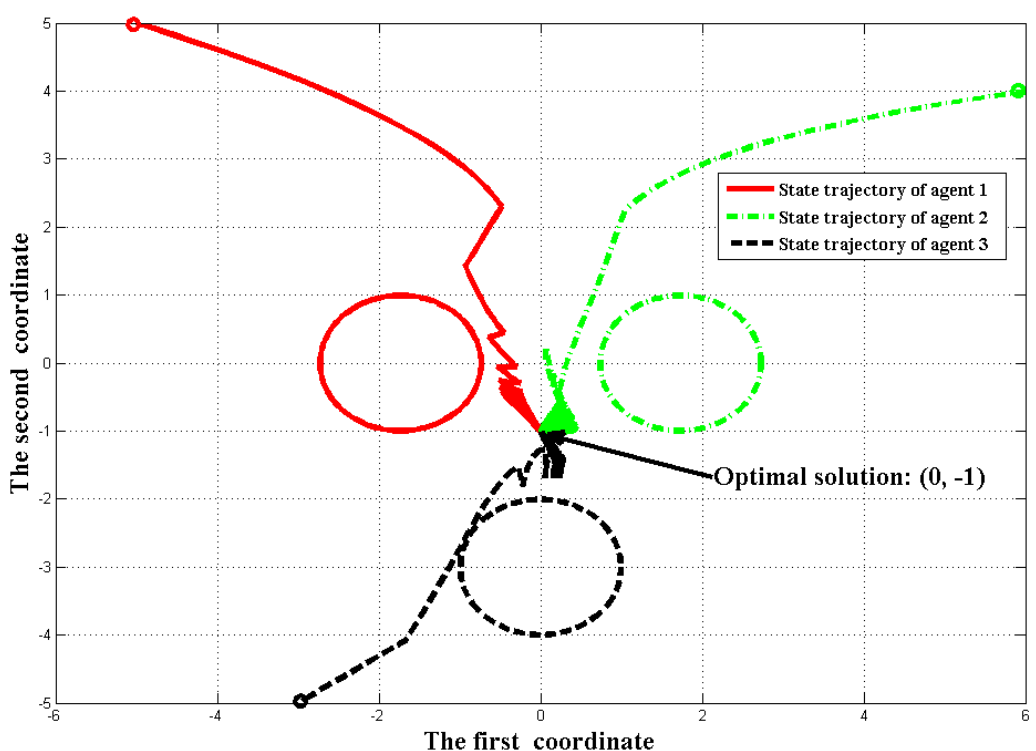

Figure 4: In the empty intersection case, all agents converge to the unique optimal solution $(0,-1)$. 
Here agents could only obtain their approximate projections and the communication graph among agents was UJSC. It was shown that the system states were always bounded for any approximate angle, and uniformly bounded for any stepsize with inferior limit greater than zero. Both nonempty intersection and empty intersection cases of convex sets were investigated with respective sufficient conditions. Moreover, the convergence error between agents' estimates and the optimal point was also obtained for the constant stepsize and approximate angle case.

\section{Acknowledgment}

The authors would like to thank Dr. Guilin Yang for discussions about geometric analysis and Mr. Peng Yi for his generous help on numerical simulations.

\section{Appendix}

Denote $\vartheta_{i}(v)=\angle\left(v-P_{X_{i}}(v), P_{X_{i}}^{a}(v)-P_{X_{i}}(v)\right)$. By Lemma $2.1(\mathrm{i}), \vartheta_{i}(v) \geq \pi / 2$ when $P_{X_{i}}^{a}(v) \neq P_{X_{i}}(v)$. In the following proofs, we omit all subscript $i$ and simplify $\theta_{i}, \vartheta_{i}, \mu_{i}$, $X_{i}$ as $\theta, \vartheta, \mu, X$.

Proof of Lemma 4.1. Let $v \in S \backslash X$ and $P_{X}^{a}(v) \neq P_{X}(v)$. We obtain

$$
\begin{aligned}
\gamma_{X}(v) & =\frac{\left|P_{X}^{h}(v)-v\right|+\left|P_{X}^{a}(v)-P_{X}^{h}(v)\right|}{\left|P_{X}^{h}(v)-v\right|} \\
& =1+\frac{\left|P_{X}^{a}(v)-P_{X}^{h}(v)\right|}{\left|P_{X}^{h}(v)-P_{X}(v)\right|} \sin \theta(v) \\
& =1+\frac{\sin \left(\vartheta(v)-\frac{\pi}{2}\right)}{\sin \mu(v)} \sin \theta(v) \\
& \leq 1+\frac{1}{\sin \mu(v)} \sin \theta(v) \\
& \leq 1+\frac{1}{\sin \mu(v)} .
\end{aligned}
$$

Then the proof is completed.

Proof of Lemma 4.2. Consider the following relation

$$
\operatorname{cone}\left(v, \mathbf{C}_{X}(v, \theta) \cap \mathbf{b}(v, X)\right)=\mathbf{C}_{X}(v, \theta)
$$

where cone $(v, M)=\{v+\lambda(z-v) \mid \lambda \geq 0, z \in M\}$ for some set $M \subseteq \mathbb{R}^{m}$. We first show the following three claims: 


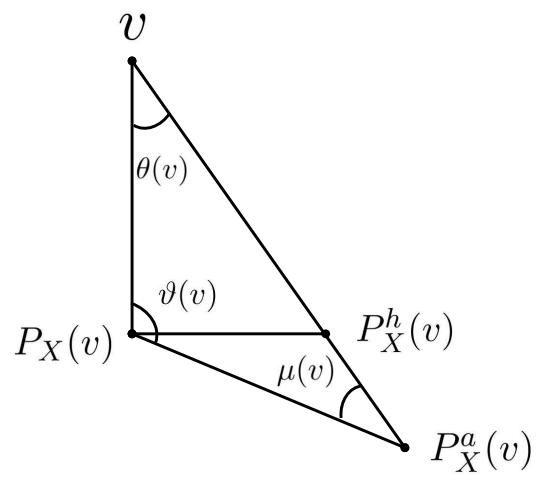

Figure 5: An illustration for the proof of Lemma 4.1.

(i) Suppose that, for any $v \in \operatorname{bd}(S)$, there is $\theta(v)>0$ such that (40) holds for $v, \theta(v)$. Then (40) holds for $\theta^{*}$ and any $v \in S \backslash X$ with sufficiently small $|v|_{X}, \theta^{*}$ is the approximate angle given in A3.

(ii) Suppose $\operatorname{int}(X) \cap \operatorname{line}\left(v, P_{X}(v)\right) \neq \emptyset$ for any $v \in \operatorname{bd}(S)$. Then the hypothesis in (i) holds.

(iii) Suppose the boundary surface of $X$ is regular. Then hypothesis in (ii) holds.

(i) is obvious. For (ii), let $z \in \operatorname{int}(X) \cap \operatorname{line}\left(v, P_{X}(v)\right)$. Then there exists $\epsilon>0$ such that $\mathbf{B}(z, \epsilon) \subseteq X$. Let $y \in \operatorname{bd}(\mathbf{B}(z, \epsilon))$ be the point for which $\angle(y-z, v-z)=\pi / 2$. Clearly, (40) holds for $v, \theta(v)$, where $\theta(v)=\angle(y-v, z-v)>0$.

We prove (iii) by contradiction. For a regular surface, its tangent plane at boundary point $z$ consists of the tangent vectors at point $z$ of all curves passing $z$. Suppose that there is $v \in \operatorname{bd}(S)$ with $\operatorname{int}(X) \cap \operatorname{line}\left(v, P_{X}(v)\right)=\emptyset$. Then, by convex set separation Theorem 11.3 on page 97 in [2], there exists a hyperplane $\mathcal{H}$ separating $X$ and line $\left(v, P_{X}(v)\right)$ properly. As a result, $\mathcal{H}$ must contain $\operatorname{line}\left(v, P_{X}(v)\right)$. Let $\mathbf{n}$ be the unit normal vector of $\mathcal{H}$ with $\angle\left(\mathbf{n}, z-P_{X}(v)\right) \geq \pi / 2$ for any $z \in X$, and $\mathcal{H}_{v}$ the tangent plane of $\operatorname{bd}(X)$ at $P_{X}(v)$. Then $\mathbf{n} \in \mathcal{H}_{v}$ since $v-P_{X}(v)$ is a normal vector of tangent plane $\mathcal{H}_{v}$. However, it is not possible that there exists a curve on $\operatorname{bd}(X)$ with tangent vector $\mathbf{n}$ at $P_{X}(v)$, which yields a contradiction.

We now show the conclusion by contradiction. Suppose that there is a sequence $\left\{v_{k}\right\}_{k \geq 0}$ with $v_{k} \in S \backslash X$ and $P_{X}^{a}\left(v_{k}\right) \neq P_{X}\left(v_{k}\right)$ such that $\lim _{k \rightarrow \infty} \mu\left(v_{k}\right)=0$. Without loss of generality, we assume $\lim _{k \rightarrow \infty} v_{k}=: v^{*} \in S \backslash \operatorname{int}(X)$.

We first consider the case of $v^{*} \in S \backslash X$. In the case of $P_{X}^{a}\left(v^{*}\right) \neq P_{X}\left(v^{*}\right)$, by the continuity we have $0=\lim _{k \rightarrow \infty} \mu\left(v_{k}\right)=\mu\left(v^{*}\right)>0$, which yields a contradiction. In the case of $P_{X}^{a}\left(v^{*}\right)=P_{X}\left(v^{*}\right)$, we have $\lim _{k \rightarrow \infty} \theta\left(v_{k}\right)=0$, which implies $\lim _{k \rightarrow \infty} \vartheta\left(v_{k}\right)=\pi$ along with $\lim _{k \rightarrow \infty} \mu\left(v_{k}\right)=0$. This, however, is impossible since the surface $\operatorname{bd}(X)$ is regular. 
We next consider the case of $v^{*} \in \operatorname{bd}(X)$. Let $r>0$ be a sufficiently small number such that (40) holds for $\theta^{*}$ and any $v+r \mathbf{n}(v)$ with $\left|v-v^{*}\right| \leq r$ and $v \in \operatorname{bd}(X)$, where $\mathbf{n}(v)$ is the unit normal vector of the tangent plane of $\operatorname{bd}(X)$ at $v$. Denote $z:=v+r \mathbf{n}(v)$. Take arbitrarily a point $\hat{y}:=\hat{y}(z) \in \operatorname{bd}\left(\mathbf{C}_{X}\left(z, \theta^{*}\right)\right) \cap \mathbf{b}(z, X) \cap \operatorname{aff}\left\{v, z, P_{X}^{a}(z)\right\}$ such that $\angle(v-z, \hat{y}-z)=\theta^{*}$. Then

$$
\mu(z) \geq \angle(v-\hat{y}, z-\hat{y}) .
$$

Moreover, it is not hard to find that, for any $z_{1}, z_{2}$ such that $z_{1} \notin X, z_{2} \notin X, P_{X}\left(z_{1}\right)=$ $P_{X}\left(z_{2}\right),\left|z_{2}\right|_{X}>\left|z_{1}\right|_{X}$, and with (40) holding for both $\left(z_{1}, \theta^{*}\right)$ and $\left(z_{2}, \theta^{*}\right)$, we have

$$
\beta\left(z_{1}\right) \geq \beta\left(z_{2}\right)
$$

where $\beta(z)=\inf _{\bar{y} \in b d\left(\mathbf{C}_{X}\left(z, \theta^{*}\right)\right) \cap \mathbf{b}(z, X)} \angle\left(P_{X}(z)-\bar{y}, z-\bar{y}\right)$.

From (41) and (42) we conclude that for any sufficiently large $k$,

$$
\mu\left(v_{k}\right) \geq \inf _{v \in b d(X),\left|v-v^{*}\right| \leq r} \beta(v+r \mathbf{n}(v))>0,
$$

which yields a contradiction. We complete the proof.

\section{References}

[1] Godsil, C., \& Royle, G. (2001). Algebraic Graph Theory. Springer-Verlag, New York.

[2] Rockafellar, R. T. (1972). Convex Analysis. New Jersey: Princeton University Press.

[3] Aubin, J., \& Cellina, A. (1984). Differential Inclusions. Berlin: Springer-Verlag.

[4] Zhang, Y., Lou, Y., Hong, Y., \& Xie, L. (2015). Distributed projection-based algorithms for source localization in wireless sensor networks. IEEE Trans. Wireless Commun., 14(6), 3131-3142.

[5] Bertsekas, D. P., \& Tsitsiklis. J. (1989). Parallel and Distributed Computation: Numerical Methods. Princeton Hall, Englewood Cliffs, NJ.

[6] Bauschke, H. (1990). Projection algorithms and monotone operators. The Thesis, Available at: http://docserver.carma.newcastle.edu.au/173/

[7] do Carmo, M. (1976). Differential Geometry of Curves and Surfaces. Prentice-Hall, Englewood Cliffs, NJ.

[8] Danskin, J. (1966). The theory of max-min, with applications. SIAM J. Applied Mathematics, 14(4), 641-664. 
[9] Pardalos, P. M., \& Romeijn. H. E. (2002). Handbook of Global Optimization. Volume 2. Kluwer Academic Publishers, London.

[10] Francis, R. L., McGinnis, L. F., \& White. J. A. (1992). Facility Layout and Location: An Analytical Approach. 2 edition. Prentice Hall, Englewood Cliffs, NJ.

[11] Deutsch, F. (1983). Rate of convergence of the method of alternating projections. in Parametric Optim. Approx., B. Brosowski and F. Deutsch, Eds. Birkhäuser, Basel, Switzerland, 76, 96-107.

[12] Gubin, L., Polyak, B., \& Raik. E. (1967). The method of projections for finding the common point of convex sets. U.S.S.R Comput. Math. Math. Phys. 7(6), 1211-1228.

[13] Lou, Y., \& Hong, Y. (2012). Target containment control of multi-agent systems with random switching interconnection topologies. Automatica, 48(5), 879-885.

[14] Lou, Y., Shi, G., Johansson, K. H., \& Hong, Y. (2013). Convergence of random sleep algorithms for optimal consensus. Systems \& Control Letters, 62(12), 1196-1202.

[15] Lou, Y., Shi, G., Johansson, K. H., \& Hong, Y. (2014). Approximate projected consensus for convex intersection computation: Convergence analysis and critical error angle. IEEE Trans. Autom. Control, 59(7), 1722-1736.

[16] Lou, Y., \& Hong, Y. (2015). Distributed surrounding design of target region with complex adjacency matrices. Available at http://arxiv.org/abs/1510.05773

[17] Shi, G., \& Johansson, K. H. (2012). Randomized optimal consensus of multi-agent systems. Automatica, 48(12), 3018-3030.

[18] Shi, G., Johansson, K. H., \& Hong, Y. (2013). Reaching an optimal consensus: Dynamical systems that compute intersections of convex sets. IEEE Trans. Autom. Control, 58(3), 610-622.

[19] Nedić, A., \& Ozdaglar, A. (2009). Distributed subgradient methods for multi-agent optimization. IEEE Trans. Autom. Control, 54(1), 48-61.

[20] Nedić, A., Ozdaglar, A., \& Parrilo, P. A. (2010). Constrained consensus and optimization in multi-agent networks. IEEE Trans. Autom. Control, 55(4), 922-938.

[21] Nedić, A., \& Ozdaglar, A. (2008). Approximate primal solutions and rate analysis for dual subgradient methods. SIAM J. Optim., 19(4), 1757-1780. 
Lou et al. Distributed Continuous-time Approximate Projection Protocols

[22] Lin, P., \& Ren, W. (2012). Distributed shortest distance consensus problem in multiagent systems. in: 51st IEEE Conference on Decision and Control, 2012, pp. 46964701.

[23] Johansson, B., Rabi, M., \& Johansson, M. (2009). A randomized incremental subgradient method for distributed optimization in networked systems. SIAM J. Optim., 20(3), 1157-1170.

[24] Hu, J., \& Feng, G. (2010). Distributed tracking control of leader-follower multi-agent systems under noisy measurement. Automatica, 46(8), 1382-1387.

[25] Shi, G., \& Hong, Y. (2009). Global target aggregation and state agreement of nonlinear multi-agent systems with switching topologies. Automatica, 45(5), 1165-1175.

[26] Shi, G., \& Johansson, K. H. (2013). Multi-agent robust consensus: Convergence analysis and application. SIAM J. Control Optim., 51(5), 3673-3691.

[27] Meng, W., Xiao, W., \& Xie, L. (2013). A projection based fully distributed approach for source localization in wireless sensor networks. Adhoc \& Sensor Wireless Networks, 18(1/2), 131-158.

[28] Lu, J., Tang, C. Y., Regier, P. R., \& Bow, T. D. (2011). Gossip algorithms for convex consensus optimization over networks. IEEE Trans. Autom. Control, 56(12), 29172923.

[29] Lu, J., \& Tang, C. Y. (2012). Zero-gradient-sum algorithms for distributed convex optimization: The continuous-time case. IEEE Trans. Autom. Control, 57(9), 23482354.

[30] Jakovetic, D., Xavier, J., \& Moura, J. M. F. (2011). Cooperative convex optimization in networked systems: Augmented lagrangian algorithms with directed gossip communication. IEEE Trans. Signal Processing, 59(8), 3889-3902.

[31] Wang, J., \& Elia, N. (2010). Control approach to distributed optimization. in: FortyEighth Annual Allerton Conference, 557-561.

[32] Wang, J., \& Elia, N. (2011). A control perspective for centralized and distributed convex optimization. in 50th IEEE Conference on Decision and Control and European Control Conference, 3800-3805.

[33] Gharesifard, B., \& Cortés, J. (2014). Distributed continuous-time convex optimization on weight-balanced digraphs. IEEE Trans. Autom. Control, 59(3), 781-786. 
[34] Gharesifard, B., \& Cortés, J. (2013). Distributed convergence to Nash equilibria in two-network zero-sum games. Automatica, 49(6), 1683-1692.

[35] Kvaternik, K., \& Pavel, L. (2012). A continuous-time decentralized optimization scheme with positivity constraints. in 51st IEEE Conference on Decision and Control, 6801-6807.

[36] Droge, G., Kawashima, H., \& Egerstedt, M. (2014). Continuous-time proportionalintegral distributed optimization for networked systems. Journal of Decision and Control, 1(3), 191-213.

YOUCHENG LOU

Department of Systems Engineering and Engineering Management

The Chinese University of Hong Kong

Shatin, N.T., Hong Kong

Academy of Mathematics and Systems Science

Chinese Academy of Sciences

Beijing 100190, China

Email: yclou@se.cuhk.edu.hk, louyoucheng@amss.ac.cn

\section{Yiguang Hong and Shouyang Wang}

Academy of Mathematics and Systems Science

Chinese Academy of Sciences

Beijing 100190, China

Email: yghong@iss.ac.cn, sywang@amss.ac.cn 